\title{
Low overhead universality and quantum supremacy using only $Z$ control
}

\author{
Brian Barch, ${ }^{1,2}$ Razieh Mohseninia $\odot,{ }^{2}$ and Daniel Lidar $\circledast^{1,2,3,4}$ \\ ${ }^{1}$ Department of Physics and Astronomy, University of Southern California, Los Angeles, California 90089, USA \\ ${ }^{2}$ Center for Quantum Information Science \& Technology, University of Southern California, Los Angeles, California 90089, USA \\ ${ }^{3}$ Department of Electrical and Computer Engineering, University of Southern California, Los Angeles, California 90089, USA \\ ${ }^{4}$ Department of Chemistry, University of Southern California, Los Angeles, California 90089, USA
}

(Received 25 March 2021; accepted 16 July 2021; published 2 September 2021)

\begin{abstract}
We consider a model of quantum computation we call "varying Z" (VZ), defined by applying controllable $Z$-diagonal Hamiltonians in the presence of a uniform and constant external $X$ field, and prove that it is universal, even in one dimension. Universality is demonstrated by construction of a universal gate set with $O(1)$ depth overhead. We then use this construction to describe a circuit whose output distribution cannot be classically simulated unless the polynomial hierarchy collapses, with the goal of providing a low-resource method of demonstrating quantum supremacy. The VZ model can achieve quantum supremacy in $O(n)$ depth in one dimension, equivalent to the random circuit sampling models despite a higher degree of homogeneity: it requires no individually addressed $X$ control.
\end{abstract}

DOI: 10.1103/PhysRevResearch.3.033207

\section{INTRODUCTION}

In the current era of noisy intermediate scale quantum computers [1], quantum architectures are limited by connectivity, gate fidelity, and various other sources of errors that limit both circuit depth and width. In response, various models of quantum computation have been developed that are designed to be relatively easy to implement on existing hardware. The strength of these models is then confirmed by demonstrating their ability to achieve universality [2-6] or quantum supremacy [7-17]. Universality is a stronger attribute, as it implies the ability to reproduce the quantum supremacy results of other models.

In this spirit, here we propose a model of quantum computation that is computationally universal even when restricted to a one-dimensional (1D) chain of qubits with only nearestneighbor interactions and a limited degree of control. This "varying-Z" (VZ) model is defined by applying a series of $Z$-diagonal (i.e., diagonal in the computational basis) Hamiltonians in the presence of a constant and homogeneous $X$ field. By homogeneous, we mean that the $X$ field is qubitpermutation invariant, requiring no individually addressed control. We consider the $\mathrm{VZ}$ model both on a general graph and in $1 \mathrm{D}$. The latter is theoretically motivated by the question of the quantum computational power of $1 \mathrm{D}$ systems $[6,18]$ and experiments with 1D systems, such as chains of fluxonium qubits [19], and chains of transmons which were used in a prequel to Google's quantum supremacy work [20]. The

Published by the American Physical Society under the terms of the Creative Commons Attribution 4.0 International license. Further distribution of this work must maintain attribution to the author(s) and the published article's title, journal citation, and DOI. general $\mathrm{VZ}$ model is physically motivated by physical systems subject to always-on transverse fields, such as superconducting flux-qubit architectures [21-24] which experience a small but always-on $X$ field in a quantum annealing context [25].

The outline of this paper is as follows: In Sec. II we review the previous results on the universality and supremacy of $1 \mathrm{D}$ models. In Sec. III we define our model and in Sec. IV we demonstrate universality by reconstructing a universal gate set. In Sec. V we apply this universality result to generate distributions capable of demonstrating quantum supremacy, and in Sec. VI we close with concluding remarks. Additional technical details are provided in the Appendices.

\section{BACKGROUND: UNIVERSALITY AND SUPREMACY VIA 1D MODELS}

Universal quantum computation is, informally, the ability to solve any problem that can be solved by any quantum computer or, more formally, the ability to approximate any unitary transformation to arbitrary accuracy in polynomial runtime [2,3]. Quantum supremacy is, also informally, the ability of a quantum computer to solve problems with a superpolynomial speedup relative to any classical computer [7]. More formally, quantum supremacy is the ability to sample from a probability distribution that cannot be efficiently approximated to arbitrary accuracy by any classical computer with access to randomness unless the polynomial hierarchy collapses, which is believed to be unlikely $[11,12]$. A number of problems exist that are solvable in polynomial time by universal quantum computers but conjectured to be impossible for classical computers to solve without a superpolynomial slowdown, so a quantum computer's universality implies its quantum supremacy $[8,10]$. A brief further discussion of the complexity basis of supremacy is provided in Appendix A. 
TABLE I. Table of various 1D models of universal quantum computation. Models are compared in terms of their requirements needed to reproduce the output distribution of a 1D quantum circuit of $n$ logical qubits and depth $d$, to within total variation distance $\epsilon$. Control requirement refers to the qubit-addressed control needed for each model. Homogeneous quantum circuits refer to quantum circuits in which every gate applied is translation invariant across all qubits, while full quantum circuits refer to circuits where gates may vary by qubit. The universality and supremacy columns give the runtime required for universality and supremacy, respectively. The models are ordered by runtime requirement.

\begin{tabular}{|c|c|c|c|c|c|}
\hline Model & $\begin{array}{c}\text { Control } \\
\text { requirement }\end{array}$ & $\begin{array}{l}\text { Physical } \\
\text { qubits }\end{array}$ & $\begin{array}{l}\text { Universality } \\
\text { runtime scaling }\end{array}$ & $\begin{array}{c}\text { Supremacy } \\
\text { runtime scaling }\end{array}$ & Reference \\
\hline QAOA & Initial state & $O[n \log (n)]$ & $O\left(n^{5} d^{5} \epsilon^{-4}\right)$ & $O\left(n^{10} \epsilon^{-4}\right)$ & {$[5]$} \\
\hline Homogeneous quantum circuits & & $4 n+2$ & $O(n d)$ & $O\left(n^{2}\right)$ & [6] \\
\hline $\mathrm{VZ}$ model & $Z$ interactions & $n$ & $O(d)$ & $O(n)$ & This work \\
\hline Full quantum circuits & $X, Z$ interactions & $n$ & $d$ & $O(n)$ & {$[14,15]$} \\
\hline
\end{tabular}

In recent years a number of complementary models of quantum computation have been proposed, with a variety of dimensionality, circuit depth, and homogeneity requirements needed to achieve universality. Here we restrict to considering models that are universal in $1 \mathrm{D}$, and compare the models on their requirements needed to reproduce a depth- $d$ universal gate set (UGS) based quantum circuit, as summarized in Table I. Let us now explain the gain achieved by the VZ model, by contrasting it with the other models featured in this table.

It is known that $1 \mathrm{D}$ gate-based quantum circuits can achieve quantum supremacy in $O(n)$ depth $[14,15]$, so we can use 1D quantum circuits as a reference point to compare the runtime requirements of other universal models in achieving quantum supremacy. One such model is the quantum approximate optimization algorithm (QAOA) [26] (briefly reviewed in Appendix B) equipped with broadcast quantum cellular automata [27], that was shown to be universal [5,28]. QAOA in $1 \mathrm{D}$ consists of a chain of qubits which undergo evolution that alternates between a homogeneous $X$ field and a potentially inhomogeneous $Z$-diagonal Hamiltonian. Cellular automata require addressed control of a single qubit, the control unit, which they use to break translational symmetry and reproduce local gates on other qubits in the chain. Using QAOA with cellular automata to reproduce the output distribution of a given 1D depth- $d$ quantum circuit to within total variation (Kolmogorov) distance $\epsilon$ requires a runtime of $O\left(n^{5} d^{5} \epsilon^{-4}\right)$ in the worst case, as shown in Appendix C.

The QAOA model requires only a fixed Z-diagonal Hamiltonian repeated with different evolution times. Alternatively, if one is capable of implementing each desired gate as an alternating sequence of homogeneous nearest-neighbor entangling gates and homogeneous local rotations, using boundary conditions of the underlying architecture to introduce spatial control, universality can be achieved in depth $O(n d)$ via the model of Ref. [6]. This model can reproduce the target circuit exactly in the absence of noise, so the time cost is independent of $\epsilon$. Compared with QAOA, this model works by applying a set of more general yet still homogeneous quantum gates.

Resourcewise, the $1 \mathrm{D} \mathrm{VZ}$ model defined here can be thought of as a midpoint between the homogenous circuit model of Ref. [6] and general 1D quantum circuits, in that it only requires individually addressed control of the $Z$ interactions. Likewise, the asymptotic depth requirement of $O(d)$ to achieve universality is between that of Ref. [6] and the original UGS-based universal quantum circuit being simulated.
While our primary concern is with universality of the $\mathrm{VZ}$ model, in Sec. V we also provide an example of a problem conjectured to be outside the complexity class SampBPP, which could be used to demonstrate quantum supremacy in a practical setting, e.g., using trapped ions or flux qubits.

\section{VARYING-Z MODEL}

We will analyze the $\mathrm{VZ}$ model from the perspective of gate layers, rather than individual gates.

Definition 1 (Gate layer). A gate layer is a depth-1 operation, equivalent to a set of gates acting on disjoint sets of qubits applied in parallel in the circuit model.

The VZ model reproduces a gate layer from a circuit in the gate model by using a series of applied layers: gate layers corresponding to the application of a single time-independent Hamiltonian, which are natural to the VZ model. We use the term effective layer to refer to the gate layer of the circuit model which is being reproduced from a set of applied layers. In the VZ model all $n$ qubits are initially prepared in the $|+\rangle^{\otimes n}$ state and then acted upon by a series of applied unitary layers. These unitaries are generated by a series of Hamiltonians composed of two terms: a $Z$-diagonal term $H_{l}^{z}$ which varies by applied layer $l$, and a homogeneous, layer-independent $X$ field $H^{x}$. Each applied layer $l$ is applied for time $t_{l}$. Note that $l$ plays the role of a discrete time index. We take the $Z$ Hamiltonians to be two-local between neighboring qubits located on the vertices $i \in \mathcal{V}$ of some underlying graph $(\mathcal{V}, \mathcal{E})$, and with interactions along the edges $(i, j) \in \mathcal{E}$ that are uniform in magnitude but can be turned on or off by edge. The $l$ th Hamiltonian may be written as

$$
\begin{aligned}
H_{l} & =H_{l}^{z}+H^{x}, \\
H^{x} & =a \sum_{i \in \mathcal{V}} X_{i}, \\
H_{l}^{z} & =b_{l} \sum_{(i, j) \in \mathcal{E}} w_{l, i j} Z_{i} Z_{j}+c_{l} \sum_{i \in \mathcal{V}} v_{l, i} Z_{i},
\end{aligned}
$$

where $w_{l, i j} \in\{0,1\}$ and $v_{l, i} \in\{0,1\}$, respectively, switch the interactions and local fields on or off for the $l$ th applied layer. We fix $a>0$ throughout the circuit, and allow $b_{l}$ and $c_{l}$ to vary by layer but not by qubit. The total number of qubits is $n=|\mathcal{V}|$. 
Definition 2 (Varying- $Z$ model). Starting from the initial state $|+\rangle \equiv|+\rangle^{\otimes n}$, apply each Hamiltonian $H_{l}$ [Eq. (1)] for corresponding time $t_{l}$, measure all the qubits in the $Z$ basis, and sample the final state.

The output probability distribution is given by

$$
P(\mathbf{s})=\left|\left\langle\mathbf{s}\left|\prod_{l} e^{-i t_{l} H_{l}}\right|+\right\rangle\right|^{2},
$$

where $\mathbf{s} \in\{0,1\}^{n}$. $^{1}$

Our main result is the following:

Theorem 1. The VZ model can simulate an arbitrary depth- $d$ quantum circuit generated by a UGS $\mathcal{G}$ to arbitrary accuracy in depth $O(d|\mathcal{G}|)$ on the same underlying graph.

Notably, any circuit generated by a size $O(1)$ UGS, e.g., the Clifford $+T$ gate set, may be simulated by the $\mathrm{VZ}$ model in depth $O(d)$. Given the universality of 1D quantum circuits with this set of gates, we have the following immediate consequence:

Corollary 1. The $1 \mathrm{D} \mathrm{VZ} \mathrm{model} \mathrm{is} \mathrm{quantum} \mathrm{computation-}$ ally universal.

The restrictions given here on the parameters $a, b_{l}, c_{l}$, and $t_{l}$ are the most restrictive for which we prove constant overhead universality. Additional spatial or temporal control would not improve runtime scaling with respect to system size, as it is already asymptotically optimal. In other words, replacing $X$-field control with a constant, always-on transverse field $H^{x}$ is sufficient for low overhead universality, as long as the $Z$-diagonal Hamiltonian can be updated between successive applied gate layers. This is clearly a significant simplification in terms of control requirements over the standard UGS approach.

Note that the initial state $|+\rangle$ is the ground state of $-H^{x}$, so it can be prepared by turning on this Hamiltonian and waiting for the system to relax into its ground state. It can also be prepared starting from the $|0\rangle^{\otimes n}$ state and applying the global Hadamard gate $W^{\otimes n}$, which is compatible with the VZ model since it requires no inhomogeneity of the $X$ field.

We remark that the VZ model resembles QAOA [26] to some extent. The main differences are the fact that in the $\mathrm{VZ}$ model the $X$ field is always on (whereas in QAOA one alternates between $H_{l}^{z}$ and $H^{x}$ ), and that in VZ model we assume that the $b$ and $c$ coefficients are $l$ dependent, whereas in QAOA they may vary by qubit but not by $l$ (see also Appendix B).

Proof outline of Theorem 1. Two-qubit gates are universal for quantum computation [3], and an arbitrary two-qubit gate can be produced with a constant number of single-qubit unitaries and $Z Z$ gates [i.e., gates generated by $Z_{i} Z_{j}$ interaction terms in the Hamiltonian, as in Eq. (1c)] [30,31]. Thus, in order to reproduce an arbitrary quantum circuit, it is sufficient to demonstrate the ability to generate arbitrary single-qubit unitaries and $Z Z$ gates. We will first demonstrate the ability

\footnotetext{
${ }^{1}$ Note that the assumption of "rectangular" pulses implied by writing $e^{-i t_{l} H_{l}}$ is, of course, experimentally unrealistic due to finitebandwidth constraints. A realistic pulse will have finite rise and fall times, but as long as the integrated pulse profile equates to $t_{l} H_{l} \forall l$, standard experimental pulse shaping methods can be used to reduce any associated systematic errors [29].
}

to reproduce layers corresponding to arbitrary single-qubit unitaries (Lemma 1) and then corresponding to $Z Z$ gates with arbitrary real coupling constants (Lemma 2). The technical challenge in proving these results is to deal with the fact the $X$ field is always on.

Each type of effective gate layer $G_{L}$ can be implemented using $l_{\max }(L) \in O(1)$ applied layers of the $\mathrm{VZ}$ model, corresponding to the decomposition

$$
G_{L}=\prod_{l=1}^{l_{\max }(L)} e^{-i t_{l}\left(H^{x}+H_{l}^{z}\right)} .
$$

As each effective layer can apply gates across all qubits in parallel, we will then analyze the decomposition of an arbitrary circuit into layers based on its UGS, and conclude that the circuit can be implemented in the VZ model with depth overhead proportional to the number of unique gates present within a single gate layer.

\section{PROOF OF UNIVERSALITY OF THE VZ MODEL}

In this section we provide a detailed proof of Theorem 1.

\section{A. Single-qubit gate layers}

Consider an effective gate layer $G_{L}$ corresponding to identical instances $g_{i}, i \in \mathcal{V}$, of an arbitrary single-qubit unitary $g$, applied to a subset of all qubits. Let $g$ apply a rotation by some angle $\gamma$ about some axis $\vec{r}=$ $[\sin (\theta) \cos (\phi), \sin (\theta) \sin (\phi), \cos (\theta)]$ of the Bloch sphere. This layer may then be decomposed as

$$
G_{L}=\bigotimes_{i}\left(g_{i}\right)^{v_{i}}=e^{-i \frac{\gamma}{2} \sum_{i} v_{i} \vec{r} \cdot \vec{\sigma}_{i}}, \quad v_{i} \in\{0,1\}
$$

for $\vec{\sigma}_{i}=\left(X_{i}, Y_{i}, Z_{i}\right)$, and $v_{i}=1$ iff unitary $g_{i}$ is applied to qubit $i$. How would we implement $G_{L}$ using just the components of the VZ model? It would appear that a simple Euler angle construction should suffice, but we explain in Appendix D why this approach fails. Instead, the following lemma provides the answer:

Lemma 1. The VZ model can implement an arbitrary effective single-qubit gate layer $G_{L}$ [Eq. (4)] in three applied layers.

Proof. We will show that $G_{L}$ may be decomposed into a product of three applied unitary layers as

$$
G_{L}=V^{\otimes n} \bigotimes_{i=1}^{n} U_{i}\left(V^{\dagger}\right)^{\otimes n}=\bigotimes_{i \in \mathcal{V}} V_{i} U_{i} V_{i}^{\dagger},
$$

with

$$
\begin{aligned}
& U_{i}=e^{-i t\left(a X_{i}+c v_{i} Z_{i}\right)}, \\
& V_{i}=e^{-i t^{\prime}\left(a X_{i}+c^{\prime} Z_{i}\right)}
\end{aligned}
$$

$V^{\dagger}$ may be implemented modulo $\pi$, and does not require changing the $X$-field strength $a$ (we suppress the $i$ subscript where convenient). The resulting effective and applied layers are depicted in Fig. 1.

Intuitively, we would like $U_{i}$ to implement a Bloch sphere rotation by $\gamma$ for qubits $i$ with $v_{i}=1$ and by 0 otherwise, up 
1

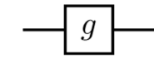

2

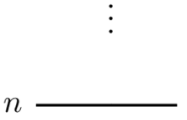

(a)
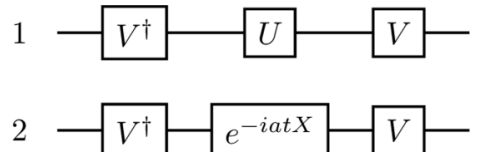

$n$

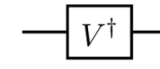

FIG. 1. (a) Example effective single-qubit gate layer $G_{L}$ in $1 \mathrm{D}$, with a gate $g$ acting on only the first qubit. (b) Implementation as applied layers in the $\mathrm{VZ}$ model. The time $t$ is chosen to make the net effect on qubits $\{2, \ldots, n\}$ the identity gate.

to equivalence modulo $2 \pi . V$ and $V^{\dagger}$ effectively rotate the rotational axis of $U_{i}$ to point along the Bloch vector $\vec{r}$.

$U_{i}$ applies a rotation of magnitude $2 \sqrt{a^{2}+\left(c v_{i}\right)^{2}} t$, so we can construct the desired $U_{i}$ by solving for $c$ and $t$ such that

$$
\begin{gathered}
2 a t=2 \pi, \quad v_{i}=0, \\
2 \sqrt{a^{2}+c^{2}} t=2 \pi+\gamma, \quad v_{i}=1 .
\end{gathered}
$$

The offset by $2 \pi$ ensures the system of equations is solvable for nonzero $t$ and real $c$. As it can be factored out as

$$
e^{-i\left(\frac{2 \pi+\gamma}{2}\right) \vec{r} \cdot \vec{\sigma}}=e^{-i \pi \vec{r} \cdot \vec{\sigma}} e^{-i \frac{\gamma}{2} \vec{r} \cdot \vec{\sigma}}=-e^{-i \frac{\gamma}{2} \vec{r} \cdot \vec{\sigma}}
$$

for unit vector $\vec{r}$, this offset's only effect on the dynamics is an overall phase. This system of equations is solved by $t=$ $\pi / a, c=(a / 2 \pi) \sqrt{(2 \pi+\gamma)^{2}-(2 \pi)^{2}}$ (recall that in the VZ model $a$ is given and fixed). For qubits with $v_{i}=0$, this choice amounts to $U_{i}=e^{-i \pi X_{i}}$ and thus no net rotation. For qubits with $v_{i}=1$ the resulting action of $U_{i}$ is

$$
\begin{aligned}
U & =e^{-i t(a X+c Z)} \\
& =e^{-i\left(\frac{2 \pi+\gamma}{2}\right)[\sin (\alpha) X+\cos (\alpha) Z],}
\end{aligned}
$$

where $\alpha=\cos ^{-1}\left(\frac{c}{\sqrt{a^{2}+c^{2}}}\right)$ [Fig. 2(a)]. Thus, the axis of rotation makes an angle $\alpha$ with the $Z$ axis.
When solving for $V$ we can ignore the $v_{i}=0$ case, as $V V^{\dagger}=I$. We choose $c^{\prime}, t^{\prime}$ in Eq. (6b) for $V$ to implement a rotation [Fig. 2(b)]:

$$
\begin{aligned}
V & =e^{-i t^{\prime}\left(a X+c^{\prime} Z\right)} \\
& =e^{-i \frac{\alpha^{\prime}}{2}[\sin (\psi) X+\cos (\psi) Z]}
\end{aligned}
$$

in order to effectively rotate the $\sin (\alpha) X+\cos (\alpha) Z$ axis into the desired axis $\vec{r}$ [Fig. 2(c)]. The necessary $\alpha^{\prime}$ and $\psi$ are solved for in Appendix E. They are

$$
\begin{aligned}
\psi & =\tan ^{-1}\left(\frac{\cos (\alpha)-\cos (\theta)}{\sin (\theta) \cos (\phi)-\sin (\alpha)}\right), \\
\alpha^{\prime} & =\sin ^{-1}\left(\frac{\sin (\theta) \sin (\phi)}{\sin (\psi-\alpha)}\right),
\end{aligned}
$$

where we take $\psi \in[0, \pi]$ and $\alpha^{\prime} \in\left[\frac{\pi}{2}, \frac{3 \pi}{2}\right]$. From here it is straightforward to solve for $c^{\prime}, t^{\prime}$.

The resulting values of $c, t, c^{\prime}, t^{\prime}$ in Eq. (6) are

$$
\begin{aligned}
t & =\frac{\pi}{a}, \\
c & =\frac{a}{2 \pi} \sqrt{(2 \pi+\gamma)^{2}-(2 \pi)^{2}}, \\
t^{\prime} & =\frac{\alpha^{\prime} \sin (\psi)}{2 a}, \\
c^{\prime} & =a \cot (\psi)
\end{aligned}
$$

for $\psi$ and $\alpha^{\prime}$ in Eq. (11). Substituting these values into Eq. (5) yields the desired $G_{L}$.

We note that in the case that $\phi=0$, Eq. (11) reduces to $\psi=(\theta+\alpha) / 2$ and $\alpha^{\prime}=\pi$. This case creates the $T$ gate when $(\theta, \gamma)=\left(0, \frac{\pi}{4}\right)$ and Hadamard gate when $(\theta, \gamma)=\left(\frac{\pi}{4}, \pi\right)$ so it is in fact sufficient to construct a universal set of single-qubit gates. However, representing an arbitrary unitary in terms of a finite-size UGS introduces a small but nonzero source of error and increased depth requirement [32]. By representing an arbitrary single-qubit unitary rather than a finite number of specific unitaries, the $\mathrm{VZ}$ model may avoid both of these setbacks.

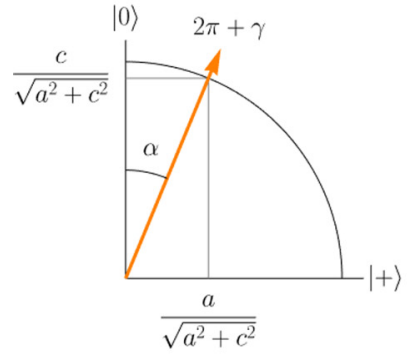

(a)

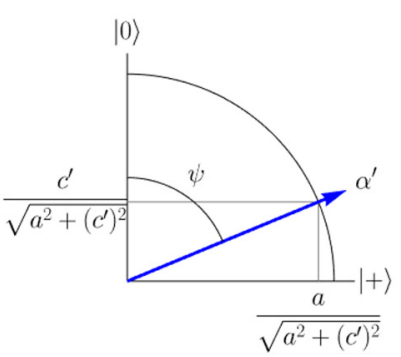

(b)

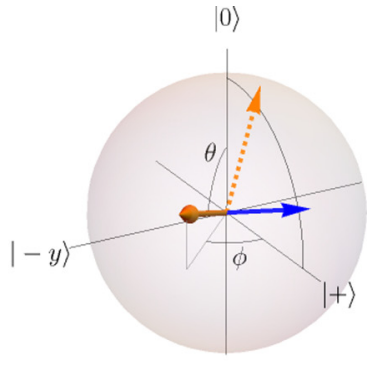

(c)

FIG. 2. (a) $U_{i}$ depicted by its rotational axis in the $X-Z$ plane of the Bloch sphere. Here $c$ is picked to make the magnitude of rotation $2 \pi+\gamma$ for qubits with $v_{i}=1$, which results in the rotational axis making an angle $\alpha$ with the $|0\rangle$ state. (b) The unitary $V$ has $t^{\prime}$ and $c^{\prime}$ such that the magnitude of rotation is $\alpha^{\prime}$ and the axis makes an angle $\psi$ with the $|0\rangle$ state for all qubits. (c) The net result of all three rotations $V U_{i} V^{\dagger}$ is that the rotational axis of $U_{i}$ (dashed orange) is rotated by $V$ (blue) to point along $\vec{r}$ (solid orange) with spherical coordinates $(\theta, \phi)$. The net rotation is of magnitude $2 \pi+\gamma$ about the axis $\vec{r}$. 


\section{B. ZZ-coupling layers}

Now consider an effective two-qubit coupling layer $G_{L}$ corresponding to $Z Z$-coupling gates $g_{i j}=e^{-i C Z_{i} Z_{j}}$ with an arbitrary real coupling constant $C>0$, and acting on some but not all pairs of qubits connected by edges in $\mathcal{E}$. Such a layer decomposes as

$$
\begin{aligned}
G_{L} & =\prod_{(i, j) \in \mathcal{E}}\left(g_{i j}\right)^{w_{i j}}, \quad w_{i j} \in\{0,1\} \\
& =e^{-i C \sum_{(i, j) \in \mathcal{E}} w_{i j} Z_{i} Z_{j}},
\end{aligned}
$$

where $w_{i j}=w_{j i}=1$ iff qubit $i$ couples to qubit $j$ and 0 otherwise. Again the question arises, how would we implement $G_{L}$ using just the components of the $\mathrm{VZ}$ model?

The restriction of Definition 1 to disjoint gates means that each qubit experiences at most a single two-qubit gate and no $>2$-qubit gates in a single time step (though we relax this restriction below, in Sec. IV D). In this case each qubit couples to at most one of its neighbors at a time:

$$
\begin{aligned}
\sum_{j} w_{i j} & =\sum_{i} w_{i j} \in\{0,1\}, \\
w_{i j} & =0 \text { for }(i, j) \notin \mathcal{E} .
\end{aligned}
$$

Then we have the following lemma:

Lemma 2. The VZ model can implement a two-qubit coupling gate layer $G_{L}$ [Eq. (13)] in at most six applied layers.

Proof. We show that this may be implemented in the VZ model using either three or six applied layers depending on whether there exist uncoupled qubits. Let $\mathcal{S} \subset \mathcal{V}$ be the set of uncoupled qubits, i.e., qubits $i$ for which $w_{i j}=0 \forall j$, and set $X_{\mathcal{S}}=\sum_{i \in \mathcal{S}} X_{i}$. The implementation takes the form

$$
G_{L}=e^{-i \frac{\nu}{2} X_{\mathcal{S}}} e^{-i t^{\prime} H^{x}} U e^{-i t^{\prime} H^{x}}
$$

for

$$
U=e^{-i t\left(H^{x}+b \sum_{(i, j) \in \mathcal{E}} w_{i j} Z_{i} Z_{j}\right)}
$$

and $e^{-i \frac{\gamma}{2} X_{\mathcal{S}}}$ an auxiliary single-qubit unitary layer, as defined in the previous subsection. This $e^{-i \frac{\gamma}{2} X_{\mathcal{S}}}$ cancels out the $X$ rotation that uncoupled qubits in $\mathcal{S}$ experience while coupled qubits are being acted upon; in the case that all qubits are coupled it reduces to the identity. The decomposition into applied layers is depicted in Fig. 3.

As shown in Appendix F, for any $C \in[0, \pi]$ the pairwise coupling condition allows us to pick parameters $b$ and $t$ such that the action of $U$ becomes

$$
\begin{aligned}
& e^{-i t\left(H^{x}+b \sum_{i j} w_{i j} Z_{i} Z_{j}\right)} \\
& \quad=e^{-i a t X_{\mathcal{S}}} e^{-i \beta X_{\overline{\mathcal{S}}}} e^{-i C \sum_{i j} w_{i j} Z_{i} Z_{j}} e^{-i \beta X_{\overline{\mathcal{S}}},}
\end{aligned}
$$

up to an overall phase, for $X_{\overline{\mathcal{S}}}=\sum_{i \notin \mathcal{S}} X_{i}$. The required $b$ and $t$ and resulting $\beta$ are solved for in Appendix F.

The effect of $\beta$ can be undone by a uniform $X$ rotation for time $a t^{\prime}=\pi-\beta$ before and after the coupling layer. This gives

$$
\begin{gathered}
e^{-i t^{\prime} H^{x}} e^{-i t\left(H^{x}+b \sum_{i j} w_{i j} Z_{i} Z_{j}\right)} e^{-i t^{\prime} H^{x}} \\
=e^{-i(a t-2 \beta) X_{\mathcal{S}}} e^{-i C w_{i j} Z_{i} Z_{j}} .
\end{gathered}
$$

In the case that $|\mathcal{S}|=0$ we have $X_{\mathcal{S}}=0$, and the result is pure $Z Z$ coupling of the desired magnitude $C$. In the case that
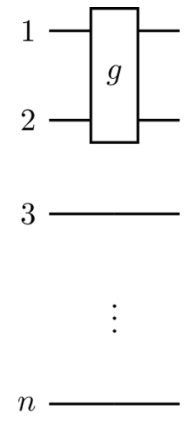

(a)
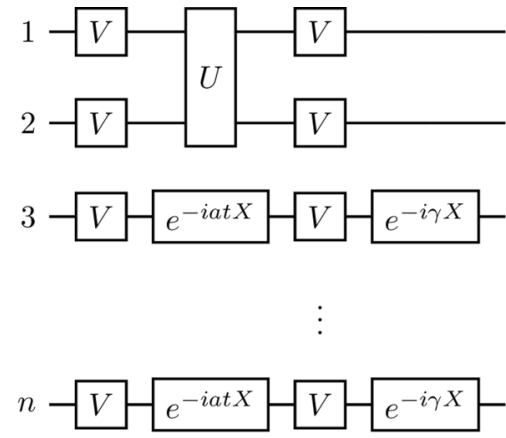

(b)
FIG. 3. (a) Example effective two-qubit gate layer $G_{L}$ with a gate $g$ acting on only the first two qubits. (b) Its implementation as applied layers in the $\mathrm{VZ}$ model. Here $V=e^{-i a t^{\prime} X}$ is applied both before and after the coupling interaction, as in Eq. (15).

some qubits are uncoupled, the extra rotation on those qubits can be undone with an auxiliary inhomogeneous $X$ rotation by angle $\gamma=4 \beta-2 a t$. In this case we have

$$
\begin{aligned}
& e^{-i \frac{\gamma}{2} X_{\mathcal{S}}} e^{-i t^{\prime} H^{x}} e^{-i t\left(H^{x}+b \sum_{i j} w_{i j} Z_{i} Z_{j}\right)} e^{-i t^{\prime} H^{x}} \\
& =e^{-i C \sum_{i j} w_{i j} Z_{i} Z_{j}} .
\end{aligned}
$$

In either case, by picking parameters

$$
\begin{aligned}
t \text { s.t. } \operatorname{sinc}(C+k \pi) & =\operatorname{sinc}\left[\sqrt{4 a^{2} t^{2}+(C+k \pi)^{2}}\right], \\
b & =(C+k \pi) t^{-1}, \\
t^{\prime} & =(\pi-\beta) a^{-1}, \\
\gamma & =4 \beta-2 a t,
\end{aligned}
$$

where $b, k, t$, and $\beta$ are solved for in Appendix $\mathrm{F}$, the effective coupling layer is implemented in at most six applied layers.

If the coupling layer is preceded or succeeded by singlequbit unitary or ZZ-coupling layers acting nontrivially on the exact same set of qubits, the required depth can be further reduced. If the temporally adjacent layer is of single-qubit unitaries, the coupling layer's required $e^{-i t^{\prime} H^{x}}$ evolution may be absorbed into the single-qubit unitary layer, and thus become effectively free, as long as the auxiliary $e^{-i \frac{\gamma}{2} X_{\mathcal{S}}}$ has $\gamma$ tuned to compensate. Furthermore, if multiple effective $Z Z$-coupling layers share a set $\mathcal{S}$ of uncoupled qubits, their auxiliary $X$ rotations will commute with all the $Z Z$ couplings, and thus can be combined into a single auxiliary unitary. Both of these methods may be used to implement SWAP gates more efficiently, as is done in Sec. V.

It is also worth mentioning that an effective layer of disjoint $e^{-i \frac{\pi}{8} Z \otimes Z}$ gates may be implemented in at most six applied layers, and one of disjoint CZ gates $e^{-i \frac{\pi}{4} Z_{1} Z_{2}} e^{-i \frac{\pi}{4}\left(Z_{1}+Z_{2}\right)}$ in at most eight by absorbing one of the $e^{-i t^{\prime} H^{x}}$ rotations into the singlequbit effective layer. Either case is sufficient for universality in combination with arbitrary single-qubit gates. Universality can also be achieved using just $C=\frac{\pi}{4}$, but the ability of the $\mathrm{VZ}$ model to apply arbitrary (real) couplings reduces depth requirements and allows exact, rather than approximate, implementation of a wider range of potential UGSs. 


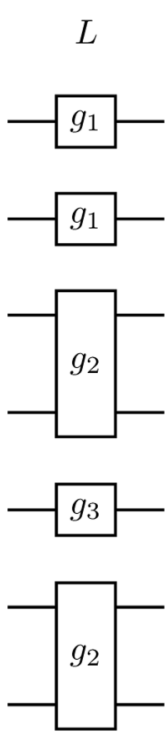

(a)

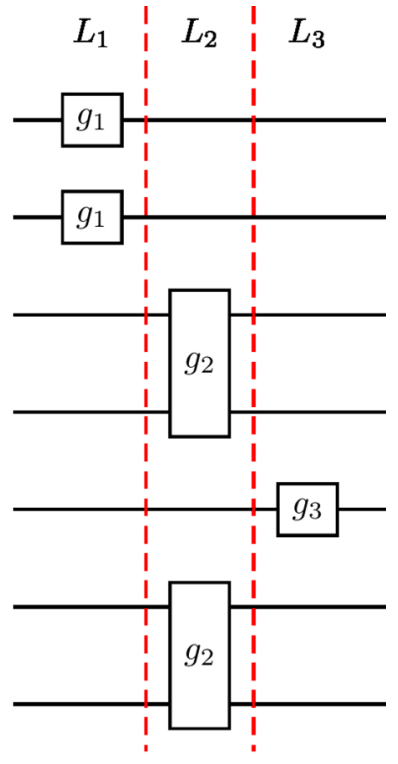

(b)
FIG. 4. (a) An example of a single effective layer $L$ of an arbitrary 1D circuit. The circuit is composed of gates $g_{l}$ of the UGS $\mathcal{G}$, which act on at most two neighboring qubits. (b) The layer $L$ implemented as three effective sublayers. Each sublayer $l$ can be written as a tensor product of $g_{l}$ or the identity across all qubits, allowing it to be implemented in the $\mathrm{VZ}$ model in constant depth using the methods of this section.

\section{Depth requirements}

Naively, a depth- $d$ quantum circuit on some graph could be implemented in the VZ model on the same graph in $O(n d)$ layers without parallelization, simply by viewing each gate as its own effective layer. While this is sufficient for universality, we can reduce the required depth by a factor of up to $n$ using parallelization in terms of a UGS, leading to Theorem 1.

Proof of Theorem 1. Say that a circuit has depth $d$ when written in terms of disjoint gates from a UGS $\mathcal{G}$, and that $\mathcal{G}$ is composed of gates that act only on nearest-neighbor qubits in some underlying architecture described by a graph $(\mathcal{V}, \mathcal{E})$. We can assume that $\mathcal{G}$ is composed of single-qubit unitaries and two-qubit $Z Z$ coupling, as any two-qubit gate can be decomposed into $O(1)$ gates of this form [30]. Then each effective layer of the original circuit can be viewed as at most $|\mathcal{G}|$ individual effective sublayers, where each sublayer requires instances of only a single type $g \in \mathcal{G}$ of nonidentity gate (Fig. 4).

After sublayer decomposition, each effective sublayer corresponds either to a single-qubit unitary or $Z Z$ coupling on monogamously paired qubits, so it is of a form that can be implemented in the VZ model in $O(1)$ applied layers. An arbitrary quantum circuit of depth $d$ can then be represented in the $\mathrm{VZ}$ model in depth $O(d|\mathcal{G}|)$ on the same graph.

For example to reproduce the effect of $\mathcal{G}=$ $\left\{W, T, e^{-i \frac{\pi}{8} Z \otimes Z}\right\}$, effective sublayers of $W$ and $T$ gates may each be implemented in three applied layers while $e^{-i \frac{\pi}{8} Z \otimes Z}$ requires up to six, so each effective layer of the original circuit requires at most 12 applied layers of the $\mathrm{VZ}$ model to implement on the same graph.
By extending the 1D implementation of arbitrary instantaneous quantum polynomial-time (IQP) circuits used in Ref. [14] to more general circuits, one sees that 1D quantum circuits are universal with $O(n)$-depth overhead. Thus, the $1 \mathrm{D} \mathrm{VZ} \mathrm{model} \mathrm{is} \mathrm{capable} \mathrm{of} \mathrm{representing} \mathrm{any} \mathrm{depth-} d$ quantum circuit expressed in terms of UGS $\mathcal{G}$ on an arbitrary graph, in depth $O(n d|\mathcal{G}|)$. Note that this $O(n)$ factor does not show up in Table I, as there the target circuit is already taken to be 1D.

The 1D case is also interesting in its own right: it is known that $1 \mathrm{D} O(n)$-depth universal quantum circuits anticoncentrate [33] and can generate distributions that cannot be efficiently classically simulated [14]. In Sec. V, we provide a circuit distribution that demonstrates this, and analyze the depth overhead required to implement it in the $\mathrm{VZ}$ model.

\section{Generalization from disjoint gates to commuting gates}

We may also extend the results above to a more general notion of gate layer, in which we weaken the Definition 1 requirement from gates being disjoint to merely commuting. That is, multiple commuting gates may act on the same qubit within a single layer. This notion of depth is the sense in which an IQP circuit is instantaneous [34].

As the decomposition in Eq. (17) required pairwise coupling, this new definition of gate layer introduces overhead proportional to the maximum degree $\Delta$ of the underlying qubit graph $(\mathcal{V}, \mathcal{E})$ in the worst case. After applying the sublayer decomposition of the previous subsection, consider an effective sublayer corresponding to a two-qubit coupling gate $g$. Within the sublayer, $g$ can act on an individual qubit $i$ once for each neighbor that qubit has. In order to restore pairwise coupling, we must further decompose into an effective sublayer for each application of $g$ on $i$ (Fig. 5). The application of a single two-qubit coupling gate layer may require as many effective sublayers as the minimum edge coloring of the underlying graph. By Vizing's theorem [35], this can be upper bounded by $\Delta+1$. In case the graph is a grid-based lattice of dimension $D$, this simply becomes the number of sublattices of paired qubits, which is $\Delta=2 D$.

With this notion of depth, a depth- $d$ quantum circuit requires a depth- $O(d|\mathcal{G}| \Delta) \mathrm{VZ}$ model implementation on the same graph. In the case of a $D$-dimensional integer lattice $\mathbb{Z}^{D}$, this becomes $O(d|\mathcal{G}| D)$. Interestingly, as a fully connected graph has $\Delta=n-1$, this notion of depth gives the $1 \mathrm{D}$ and same graph $\mathrm{VZ}$ models the same asymptotic depth scaling in the worst case.

\section{DEMONSTRATING QUANTUM SUPREMACY}

\section{A. Homogeneous $X$ field}

In this section we apply the previous results towards demonstration of quantum supremacy.

Corollary 2. Given certain standard complexity theoretic assumptions, executing an $O(n)$-depth $1 \mathrm{D} \mathrm{VZ} \mathrm{model} \mathrm{circuit}$ can generate samples from a distribution that cannot be approximately simulated in poly $(n)$ time by a classical computer in the worst case.

The proof follows from Theorem 1 and the distribution generated in Refs. [8,14], in which it is shown that no classical computer can efficiently sample from a distribution approxi- 


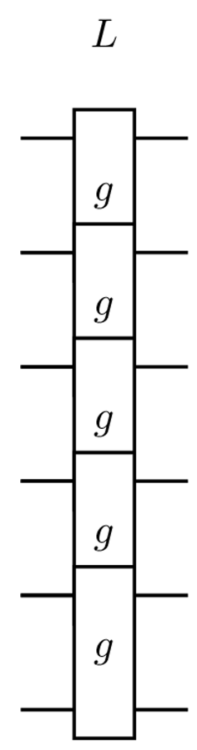

(a)

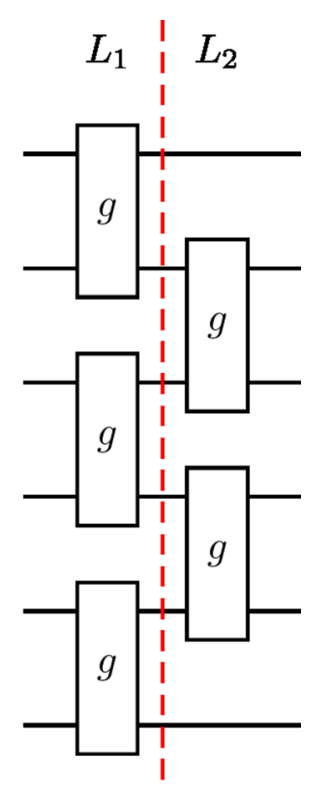

(b)
FIG. 5. (a) An example of an effective coupling sublayer $L$ of a 1D circuit, in which the coupling gate $g$ is applied across all qubits, rather than restricted to pairwise coupling. (b) The sublayer $L$ implemented as two effective coupling sublayers, each with pairwise coupling.

mating the one in question unless the polynomial hierarchy collapses, which is believed to not be the case (detailed in Appendix A). Here we describe the distribution and account for the exact overhead introduced from generating it in the $\mathrm{VZ}$, rather than gate-based, model of quantum computation. This distribution was proposed in Ref. [8] and is the output distribution of an IQP model with long-range interactions:

$$
P_{\mathrm{IQP}}(\mathbf{s})=\left\langle\mathbf{s}\left|W^{\otimes n} e^{-i C_{z}}\right|+\right\rangle,
$$

where

$$
C_{z}=w_{i j} Z_{i} Z_{j}+v_{i} Z_{i}, \quad w_{i j}, v_{i} \in\left\{\frac{1}{8} k \pi\right\}_{k=0}^{7},
$$

with $w_{i j}$ and $v_{i}$ chosen uniformly at random from their domains and $\mathbf{s}$ a string in the $Z$ basis.

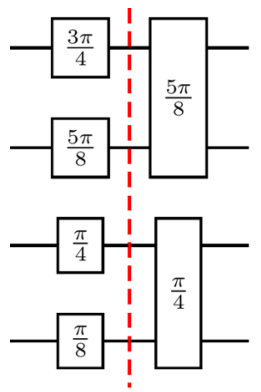

(a)

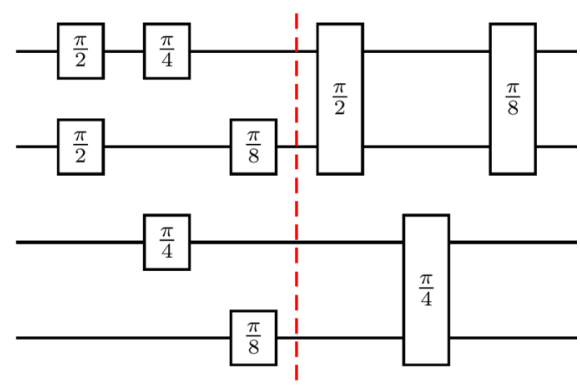

(b)
FIG. 7. (a) The first two layers of an example circuit with arbitrarily chosen $w_{i j}$ and $v_{i}$. Z-diagonal gates are denoted by their degree of rotation. (b) The same two layers rewritten as effective sublayers which can be implemented in the $\mathrm{VZ}$ model. For example, the gate corresponding to $v_{2}=\frac{5 \pi}{8}$ in (a) is decomposed into $\frac{\pi}{2}$ and $\frac{\pi}{8}$ gates in (b), with the $\frac{\pi}{8}$ gate shifted so that it is executed in parallel with the other $\frac{\pi}{8}$ gate from (a) to form a sublayer.

In the $\mathrm{VZ}$ model we can generate the distribution of Eq. (21) using a modified version of the circuit design of Ref. [14], as is depicted in Fig. 6. The depth overhead of implementing this circuit in the $\mathrm{VZ}$ model is affected by choice of UGS. Rather than implementing each term in $C_{Z}$ as an independent single-qubit or coupling gate [Fig. 7(a)], we break each layer of single-qubit and coupling gates into three effective sublayers by using the binary decomposition

$$
v_{i}=\frac{4 \pi}{8} a_{i}+\frac{2 \pi}{8} b_{i}+\frac{\pi}{8} c_{i}, \quad a_{i}, b_{i}, c_{i} \in\{0,1\}
$$

and implementing each $a, b, c$ as its own sublayer. Each $Z Z$-coupling layer is decomposed into three sublayers with an equivalent decomposition on $w_{i j}$ [Fig. 7(b)]. A SWAP gate may be implemented by decomposition into a product of Hadamard gates and single-qubit and two-qubit $Z$-diagonal gates (Fig. 8).

Using the methods of Sec. III, each sublayer of Fig. 7(b) corresponding to a single-qubit gate can be implemented in three applied layers, and thus the set of all single-qubit gates requires nine applied layers using the decomposition of Eq. (23). Similarly, each coupling layer requires 18 applied

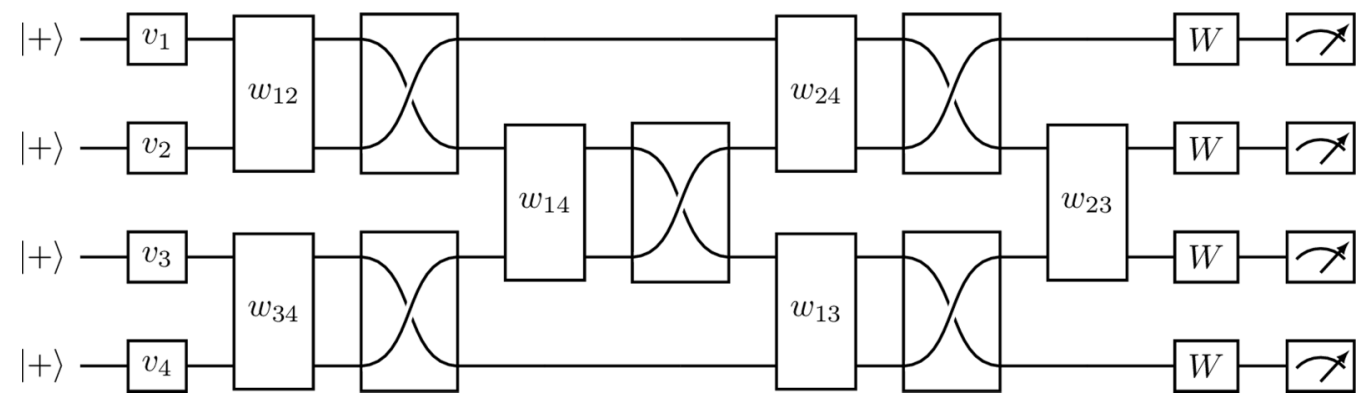

FIG. 6. The $n=4$ case of a $1 \mathrm{D} O(n)$-depth circuit capable of generating the distribution in Eq. (21) via alternating layers of $Z Z$ coupling and SWAP gates. It is equivalent to that of Ref. [14], but composed of Hadamard gates $(W)$, SWAP gates, and $Z$-diagonal gates of the form $e^{i v_{i} Z_{i}}$ and $e^{i w_{i j} Z_{i} Z_{j}}$, denoted by their degree of rotation $v_{i}$ or $w_{i j}$. In this design qubits effectively act as particles moving past each other in $1 \mathrm{D}$, and after $n$ layers each qubit has a chance to interact with each other qubit once. As $Z$-diagonal gates commute, multiple interactions between any pair of qubits can be implemented as a single interaction. Thus, this $1 \mathrm{D}$ depth- $O(n)$ circuit is sufficient to implement the circuit implied by Eq. (21). 


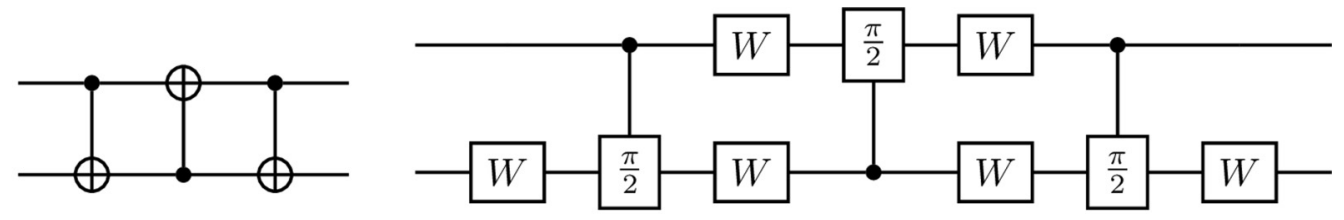

(a)

(b)

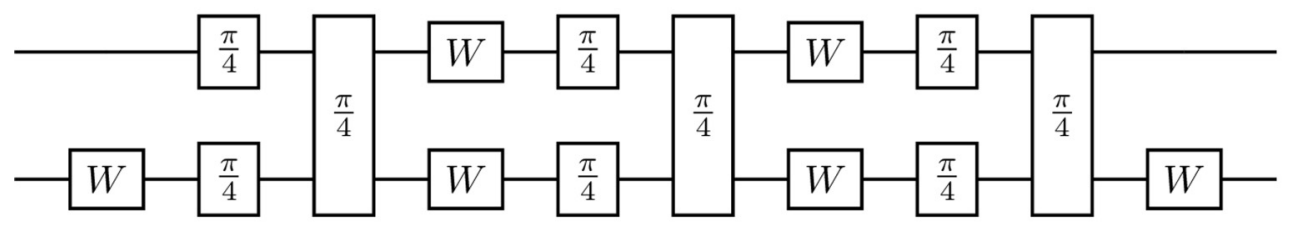

(c)

FIG. 8. The SWAP gate represented as (a) a product of CNOT gates, (b) Hadamard (W) and CZ gates, and (c) Hadamard, $e^{-i \frac{\pi}{4} Z}$, and $e^{-i \frac{\pi}{4} Z \otimes Z}$ rotation gates. The latter may be implemented in the VZ model using the methods of Sec. III.

layers, or $18 n$ in total between the $n Z Z$-coupling layers. In implementation of the SWAP layer [Fig. 8(c)], we combine adjacent single-qubit unitary layers acting nontrivially on the same qubits. The result is that a SWAP gate requires only five effective single-qubit unitary layers, or 15 applied layers. We further combine the $e^{-i t^{\prime} H^{x}}$ rotations needed for coupling layers into the adjacent single-qubit unitary layers; all but one $e^{-i t^{\prime} H^{x}}$ rotation can be absorbed this way. All the auxiliary $e^{-i \frac{\gamma}{2} X_{\mathcal{S}}}$ rotations can be combined, so the set of three effective $Z Z$-coupling layers requires only four applied layers to implement if every qubit is coupled $(|\mathcal{S}|=0)$, or seven otherwise. In total, each of the $n$ effective SWAP layers requires either 19 or 22 applied layers, depending on whether the SWAP gates span every qubit or not. When $n$ is even, half of the SWAP layers act on all qubits, but for odd $n$ every layer has uncoupled qubits, so in general the total overhead for the SWAP gate layers is at most $22 n$. Summing the $18 n$ applied layers for $Z Z$ coupling, $22 n$ applied layers for SWAP gates, nine applied layers for single-qubit gates, and final all-qubit Hadamard, the entire circuit can be implemented in $40 n+10$ applied layers.

These calculations assume a noiseless computation model. The presence of noise can reduce the fidelity of the output distribution of a circuit to the point that it no longer demonstrates quantum supremacy (see, e.g., Ref. [17] for a discussion of this in the context of random circuit sampling). However, in the case that each output bit has $O(1)$ chance of error (due to measurement error, depolarizing noise, etc), for the distribution discussed here even a classical repetition code with $O[\ln (n)]$ repetitions is sufficient to regain the fidelity needed to demonstrate supremacy [34]. The universality of the $\mathrm{VZ}$ model allows it to perform this repetition code efficiently, in contrast to nonuniversal methods of demonstrating quantum supremacy, which are not necessarily able to correct for the effects of decoherence.

\section{B. Alternating $X$ field}

In this section we consider a slight modification of the VZ model in which the $X$ field can be turned off for half the qubits at a time, either those of even or odd index. This modification reduces the depth required to apply the SWAP gate while still being easier to implement than the fully inhomogeneous circuit model, and thus reduces the total depth required to demonstrate quantum supremacy. We still use the homogeneous $X$-field method for implementing the $Z Z$ coupling layers. For even $n$, in each SWAP layer the SWAP gates alternate between acting pairwise on one sublattice such that they cover all of the qubits, and acting pairwise on the other sublattice such that there are two unaffected qubits at the boundaries of the qubit chain. For odd $n$ every sublattice acted on by SWAP gates has a single unaffected qubit. Cases with unaffected qubits require more layers to cancel the $X$ rotations these boundary qubits experience while the other qubits are being swapped, as discussed below.

In the alternating $X$-field method, instead of implementing the SWAP gate as three CNOT gates as in Fig. 8(a), we use different building blocks: $Z Z$-coupling layers with nonzero $X$ fields on either only the even or only the odd qubits, as shown in Fig. 9. In Appendix $G$ we show that in this construction a SWAP gate layer spanning all qubits can be implemented in seven applied layers. In the case of unswapped qubits, these qubits experience a different net $X$ rotation depending on whether they are of even or odd qubit index. As there are at most two values of unwanted $X$ rotation after the SWAP layer, these can be undone in at most two single-qubit effective layers, or six applied layers. This brings the total number of applied layers to implement a single effective SWAP layer to seven if every qubit is swapped, 10 if there is a single unswapped qubit, or at most 13 otherwise.

For even $n$ the model alternates between SWAP layers with zero and two unswapped qubits, so the set of all SWAP layers requires $7 \frac{n}{2}+13 \frac{n}{2}=10 n$ applied layers. For odd $n$, every SWAP layer has a single unswapped qubit, so the SWAP layers

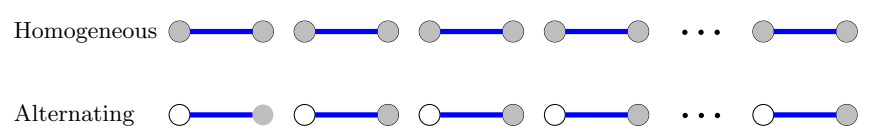

FIG. 9. Homogeneous vs alternating pattern of $X$ fields. Blue lines represent $Z Z$ couplings, which form a sublattice. Gray circles represent nonzero $X$ field, while white circles have no $X$ field. 
require a total of $10 n$ applied layers, the same as in the even $n$ case. Including $18 n$ applied layers of $Z Z$-coupling gates and 10 of single-qubit gates and Hadamards, the total number of applied layers required to reach supremacy becomes $28 n+10$. This shows a cost reduction from $40 n+10$ (in the case of a fully homogeneous $X$ field) to $28 n+10$ (in the case of an alternating $X$-field model).

\section{CONCLUSIONS}

Minimalism is both a sound engineering design principle and a desirable feature of theoretical models. By minimalism we mean a reduction in the consumption of some or more precious resources, or the reliance on a small set of assumptions. This work is an attempt to be minimalistic about the resources and assumptions underlying both implementations and models of universal quantum computation and quantum supremacy. From a practical perspective, spatially homogeneous or global control can be a significant advantage over individualized or local control since the latter typically involves additional control wires or lasers, each of which is another source of noise and decoherence. From a theoretical perspective, it is interesting to investigate the computational power of fully or partially translationally invariant models in low dimensions.

In accordance with this perspective we have studied here the power of a minimalistic model that assumes spatially homogeneous $X$-field control but spatially inhomogeneous $Z$-field control [Eq. (1)]. We have shown that this "VZ model" can be used to demonstrate quantum supremacy even in $1 D$, and is furthermore universal for quantum computation. The overhead required to implement the circuit model with a standard universal gate set using the $1 \mathrm{D} \mathrm{VZ}$ model is constant in system size and is significantly lower than the universal version of QAOA (see Table I). We are unaware of any model of quantum computation with a higher degree of homogeneity that achieves the same asymptotic depth scaling as the $\mathrm{VZ}$ model.

Perhaps the most immediate application of the VZ model would be a demonstration of quantum supremacy in the IQP setting, which we have shown here can be implemented in at most $40 n+10$ layers of control pulses, for a circuit of width $n$. This model may be the next to be used for a quantum supremacy demonstration, now that this has been done using random circuit sampling [16] and boson sampling [36]. Such a demonstration within the VZ model would be particularly viable using flux qubits [22-24], where leaving a constant and homogeneous $X$ field on while locally controlling only $Z$ fields and interactions is both simpler and less prone to noise than also controlling the $X$ field.

\section{ACKNOWLEDGMENTS}

We thank P. Zanardi for many insightful discussions. This research was sponsored by the Army Research Office and was accomplished under Grant No. W911NF-20-1-0075. This research is also based upon work (partially) supported by the Office of the Director of National Intelligence (ODNI), Intelligence Advanced Research Projects Activity (IARPA), and the Defense Advanced Research Projects Agency (DARPA), via the US Army Research Office Contract No. W911NF17-C-0050. The views and conclusions contained herein are those of the authors and should not be interpreted as necessarily representing the official policies or endorsements, either expressed or implied, of the ODNI, IARPA, DARPA, ARO, or the US Government. The US Government is authorized to reproduce and distribute reprints for Governmental purposes notwithstanding any copyright annotation thereon. This work is partially supported by a DOE/HEP QuantISED program grant, QCCFP/Quantum Machine Learning and Quantum Computation Frameworks (QCCFP-QMLQCF) for HEP, Grant No. DE-SC0019219.

\section{APPENDIX A: COMPLEXITY BASIS OF QUANTUM SUPREMACY}

We begin with some terminology. A decision problem is a problem where, given an input string, a computer is asked to answer yes or no. A sampling problem is a problem where a computer is asked to sample strings from some distribution, either exactly or approximately to within some error bound. Bounded-error probabilistic polynomial-time (BPP) is the computational complexity class of decision problems that a classical computer, with access to true randomness, can solve with an error probability less than $\frac{1}{3}$ for all instances in time polynomial in input size. It can be viewed as the class of decision problems that it is realistic for a classical computer to solve. Bounded-error quantum polynomial-time (BQP) is the equivalent class for quantum computers. The corresponding classes of approximate and exact sampling problems are SampBPP and ExactSampBPP for classical computers and SampBQP and ExactSampBQP for quantum.

It is widely believed that ExactSampBQP $\neq$ ExactSampBPP, due to a hypothetical tool called postselection [37]. Postselection is the ability to select from only samples that fit some criteria, effectively selecting the results of randomness after all computation is done. The classes of decision problems associated with BPP and BQP when the computer has access to postselection are referred to as PostBPP and PostBQP. PostBPP is known to be within the third level of the polynomial hierarchy, a hierarchy of classes of decision problems representing a generalization of the P vs NP distinction. However, it is known by Toda's and Aaronson's theorems that the entire polynomial hierarchy has a polynomial-time reduction to PostBQP, i.e., that given the ability to efficiently solve any individual problem in PostBQP, one could efficiently solve every problem in the polynomial hierarchy $[11,37,38]$. If ExactSampBQP $=$ ExactSampBPP, then PostBQP $=$ PostBPP, and the entire polynomial hierarchy would be contained within its third level, a situation referred to as collapse of the polynomial hierarchy. This is conjectured to not happen for the same reason it is believed that $\mathrm{P} \neq \mathrm{NP}$.

Verifying that one has solved a problem in ExactSampBQP requires knowing the distribution one's quantum device is sampling from. Because in reality the number of samples one can take is always finite, this distribution cannot be perfectly calculated. Instead we must address approximate sampling problems (in the class SampBQP) and either show or conjecture that those are as hard as the corresponding problems in ExactSampBQP. Verifying that what one can realistically 
sample is still computationally hard often relies on anticoncentration of the output distribution [13,34]: the outcome probabilities must be sufficiently spread across all possible outcomes of measurements, so that there is a nonvanishing signal-to-noise ratio for at least a constant fraction of all possible outcomes.

\section{APPENDIX B: QAOA VS THE VZ MODEL}

In QAOA [26] one considers a Hamiltonian of the form

$$
\begin{aligned}
H(t) & =a(t) H^{X}+d(t) H^{Z}, \\
H^{X} & =\sum_{i \in \mathcal{V}} X_{i}, \\
H^{Z} & =\sum_{(i, j) \in \mathcal{E}} b_{i j} Z_{i} Z_{j}+\sum_{i \in \mathcal{V}} c_{i} Z_{i},
\end{aligned}
$$

for $n$ qubits occupying the vertices $\mathcal{V}$ of a graph $\mathcal{G}=\{\mathcal{V}, \mathcal{E}\}$. The parameters $b_{i j}$ and $c_{i}$ are controllable longitudinal local field and coupling constants, respectively, and $a(t)$ and $d(t)$ are time-dependent control functions. Note that $H_{l}$ [Eq. (1)] is an instance of $H(t)$, for each fixed $l$ and $t$.

The level- $p$ QAOA produces an approximation $C_{p}^{*}$ to the optimal value of the classical cost function represented by $H^{Z}$ :

$$
\begin{aligned}
U(\beta, \gamma) & =e^{-i \beta H^{X}} e^{-i \gamma H^{Z}}, \\
|\psi(\boldsymbol{\gamma}, \boldsymbol{\beta})\rangle & =\left(\prod_{k=1}^{p} U\left(\beta_{k}, \gamma_{k}\right)\right)|+\rangle, \\
C_{p}^{*} & =\min _{\boldsymbol{\gamma}, \boldsymbol{\beta}}\left\langle\psi(\boldsymbol{\gamma}, \boldsymbol{\beta})\left|H^{Z}\right| \psi(\boldsymbol{\gamma}, \boldsymbol{\beta})\right\rangle,
\end{aligned}
$$

where $\boldsymbol{\gamma}=\left(\gamma_{1}, \ldots, \gamma_{p}\right), \boldsymbol{\beta}=\left(\beta_{1}, \ldots, \beta_{p}\right)$ are the angles that parametrize the circuit. Various heuristic methods for choosing these angles have been considered, and for small values of $p=O(1)$ the optimization can be done exactly [39].

The VZ model (Definition 2) differs from QAOA in that the alternating sequence of unitaries always includes $H^{X}$, unlike the QAOA sequence given in Eq. (B2a). It also differs in that in QAOA the $H^{Z}$ Hamiltonian is fixed, while in the $\mathrm{VZ}$ model we assume that the $b, c, w$, and $v$ coefficients in Eq. (1) are controllable from layer to layer, which would be equivalent to making $b$ and $c$ time dependent in Eq. (B1c).

\section{APPENDIX C: DEPTH SCALING OF QAOA UNIVERSALITY}

Here we analyze the required depth of the QAOA model used in Ref. [5] to reproduce the output of a $1 \mathrm{D}$ circuit to within a given total variation distance $\epsilon$. This model of QAOA uses coirrationality of terms in a $Z$-diagonal Hamiltonian $H_{Z}$ to reproduce a translationally invariant gate layer $U$ composed of a tensor product of one to two qubit gates across all qubits. The evolution time needed to reproduce a specific gate layer such that

$$
\left\|e^{-i t H_{Z}}-U\right\| \leqslant \epsilon^{\prime}
$$

is $t \in O\left(\epsilon^{\prime-4}\right)$, where $\|\cdot\|$ is the operator norm.

The cellular automata method applies individually addressed gates by using SWAP gates to effectively walk a certain qubit, dubbed the control unit, across the chain until it is adjacent to a qubit being acted on [27], then performs controlled gates. It thus requires $O(n)$ depth to implement a single gate. Assuming the ability to parallelize, a $1 \mathrm{D}$ circuit of depth $d$ with $O(n d)$ gates will then require $O(n d)$-depth cellular automata to reproduce. In the QAOA implementation this becomes $O\left(n d \epsilon^{\prime-4}\right)$, but we would like to bound runtime in terms of total variation distance $\epsilon$ of the output distribution, not operator norm error $\epsilon^{\prime}$ of each individual gate layer.

Define an $n d$-layer cellular automata protocol as the application of a series of gates $\left\{U_{l}\right\}_{l=1}^{\text {nd }}$ to some initial state $\rho_{0}$, with $\rho_{l}$ the state of the system after application of $U_{l}$. Let the implementation of this gate in QAOA be composed of gate layers $U_{l}^{\prime}=U_{l}+\delta_{l}$ for gate error $\delta_{l}$ with $\left\|\delta_{l}\right\|<\epsilon^{\prime}$, and resulting states $\rho_{l}^{\prime}$. We can bound the trace distance $\left\|\rho_{l}^{\prime}-\rho_{l}\right\|_{1}$ between the ideal and QAOA implementations of the circuit in terms of that of $\left\|\rho_{l-1}^{\prime}-\rho_{l-1}\right\|_{1}$ as

$$
\begin{aligned}
\left\|\rho_{l}^{\prime}-\rho_{l}\right\|_{1}= & \left\|U_{l}^{\prime} \rho_{l-1}^{\prime} U_{l}^{\prime \dagger}-U_{l} \rho_{l-1} U_{l}^{\dagger}\right\|_{1} \\
= & \| U_{l}^{\prime}\left(\rho_{l-1}^{\prime}-\rho_{l-1}\right) U_{l}^{\prime \dagger}+U_{l}^{\prime} \rho_{l-1} U_{l}^{\prime \dagger} \\
& -U_{l} \rho_{l-1} U_{l}^{\dagger} \|_{1} \\
\leqslant & \left\|\rho_{l-1}^{\prime}-\rho_{l-1}\right\|_{1}+\| U_{l}^{\prime} \rho_{l-1} U_{l}^{\prime \dagger} \\
& -U_{l} \rho_{l-1} U_{l}^{\dagger} \|_{1}
\end{aligned}
$$

by using the triangle inequality and unitary invariance of the trace norm. Using submultiplicativity and the fact $\left\|\rho_{l-1}\right\|_{1}=$ 1, we can further bound the right-hand side:

$$
\begin{aligned}
& \left\|U_{l}^{\prime} \rho_{l-1} U_{l}^{\prime \dagger}-U_{l} \rho_{l-1} U_{l}^{\dagger}\right\|_{1} \\
& \quad=\left\|\left(U_{l}+\delta_{l}\right) \rho_{l-1}\left(U_{l}+\delta_{l}\right)^{\dagger}-U_{l} \rho_{l-1} U_{l}^{\dagger}\right\|_{1} \\
& \quad \leqslant\left\|\rho_{l-1} \delta_{l}^{\dagger}\right\|_{1}+\left\|\delta_{l} \rho_{l-1}\right\|_{1}+\left\|\delta_{l} \rho_{l-1} \delta_{l}^{\dagger}\right\|_{1} \\
& \quad \leqslant 2\left\|\delta_{l}\right\|\left\|\rho_{l-1}\right\|_{1}+\left\|\delta_{l}\right\|^{2}\left\|\rho_{l-1}\right\|_{1} \\
& \quad \leqslant 2 \epsilon^{\prime}+\epsilon^{\prime 2}
\end{aligned}
$$

where we used $\left\|A^{\dagger}\right\|=\|A\|$ and $\|A B\|_{1} \leqslant\|A\|\|B\|_{1}$ for any pair of operators $A$ and $B[40,41]$. Summarizing, we have

$$
\left\|\rho_{l}^{\prime}-\rho_{l}\right\|_{1}-\left\|\rho_{l-1}^{\prime}-\rho_{l-1}\right\|_{1} \leqslant 2 \epsilon^{\prime}+\epsilon^{\prime 2}, \quad \forall l .
$$

For a circuit of $n d$ gate layers, the total variation distance between the output distributions of the ideal and QAOA implementations can then be bounded as

$$
\begin{aligned}
\sum_{s}\left|P^{\prime}(s)-P(s)\right| & \leqslant\left\|\rho_{n d}^{\prime}-\rho_{n d}\right\|_{1} \\
& \leqslant 2 n d \epsilon^{\prime}+n d \epsilon^{\prime 2},
\end{aligned}
$$

where $s$ represents a bit string, the first inequality comes from bounding the total variation distance by the trace-norm distance, ${ }^{2}$ and the second by recursively applying Eq. (C4) and using the fact that $\rho_{0}^{\prime}=\rho_{0}$.

\footnotetext{
${ }^{2} \sum_{s}\left|P^{\prime}(\boldsymbol{s})-P(\boldsymbol{s})\right| \leqslant \operatorname{Tr}\left|\rho^{\prime}-\rho\right|$, where $P(\boldsymbol{s})=\operatorname{Tr}(|\boldsymbol{s}\rangle\langle\boldsymbol{s}| \rho)$, with $\rho=C|0\rangle\langle 0| C^{\dagger}$, and the inequality follows since the trace-norm distance is the maximum of $\frac{1}{2} \sum_{s}\left|\operatorname{Tr}\left[E_{s}\left(\rho^{\prime}-\rho\right)\right]\right|$ over all possible generalized measurements $E_{s}$, which includes the projective measurement $|\boldsymbol{s}\rangle\langle\boldsymbol{s}|$ [42].
} 
Suppose we wish to approximate a circuit to total variation distance $O(\epsilon)$. Then assuming the worst case bound in Eq. (C5), we must have $\epsilon^{\prime}=O\left(\frac{\epsilon}{n d}\right)$. In this case the total runtime of the QAOA circuit is $O\left(n d \epsilon^{\prime-4}\right)=O\left(n^{5} d^{5} \epsilon^{-4}\right)$. Note that in Eq. (C1) we used the operator norm rather than the trace norm as in Ref. [5]. The reason is that the trace norm is extensive in the Hilbert space dimension, meaning that in the worst case it is $2^{n+1}$ times larger than the operator norm for the difference between two unitaries as in Eq. (C1). Had we used the trace norm, then, since the first $n$ in Eq. (C5b) would have been modified by the same factor, we would have found the runtime to be larger by the fourth power of the same factor than if we use the operator norm.

\section{APPENDIX D: FAILURE OF THE EULER ANGLES CONSTRUCTION TO GENERATE SINGLE-QUBIT GATES IN THE VZ MODEL}

We can define su(2) generators in the VZ model:

$\tilde{X}=\cos (\alpha) X+\sin (\alpha) Z, \quad \tilde{Z}=-\sin (\alpha) X+\cos (\alpha) Z$.

(D1)

It is simple to check that this pair satisfies the su(2) commutation relations along with $Y$ (e.g., $[\tilde{Z}, \tilde{X}]=2 i Y$, etc.). From here we can construct any $\mathrm{SU}(2)$ single-qubit gate using the standard Euler angles construction:

$$
\begin{aligned}
g(\phi, \theta, \psi) & =U^{z}(\phi) U^{x}(\theta) U^{z}(\psi) \\
& =\left[\begin{array}{cc}
\cos (\theta) e^{-i(\psi+\phi)} & -i \sin (\theta) e^{-i(\phi-\psi)} \\
-i \sin (\theta) e^{i(\phi-\psi)} & \cos (\theta) e^{i(\psi+\phi)}
\end{array}\right],
\end{aligned}
$$

where the angles take values within the intervals $\theta \in[0, \pi / 2]$, $\phi \in[0, \pi], \quad \psi \in[0,2 \pi](\bmod \pi)$, and where $U^{x}(\varphi)=$ $\exp (-i \varphi \tilde{X})$ and $U^{z}(\varphi)=\exp (-i \varphi \tilde{Z})$. This approach is certainly feasible for an applied layer in which all qubits undergo the same single-qubit gate. However, it fails when at least one qubit (but not all) in an applied layer is idle, since in the Euler angle construction the only way to generate the identity gate is to choose the angles as

$$
\{\theta=0, \psi=2 \pi-\phi\} \quad \bmod \pi .
$$

The problem is that fixing $\theta=0$ restricts the ability to generate an arbitrary single-qubit gate on the nonidle qubits, i.e., suppose that the applied layer includes one idle and another nonidle qubit requiring the pure $\tilde{X}$ rotation $g_{2}(0, \theta, 0)=$ $g(\phi, \theta, 2 \pi-\phi)$ with $\theta>0$. It is not possible to implement both gates without restricting generality within the same time interval (applied layer) since this limits the allowed values of $\theta$. Namely, for a layer of duration $t$, on the one hand we would need $t \cos \alpha=k \pi$ and $t \sin \alpha=k \pi$ for the idle qubit (using $\theta=k \pi$ with integer $k$ instead of $\theta=0$ ), but on the other hand we would also need $t \cos \alpha=\theta$ and $t \sin \alpha=\theta$ for the nonidle qubit, thus forcing $\theta$ to be a multiple of $\pi$.

\section{APPENDIX E: PROOF OF EQ. (11)}

In this Appendix our goal is to show that we can decompose a desired arbitrary single-qubit rotation

$$
g=e^{-i \frac{\gamma^{\prime}}{2} \vec{r} \cdot \vec{\sigma}},
$$

where $\vec{r}=[\sin (\theta) \cos (\phi), \quad \sin (\theta) \sin (\phi), \quad \cos (\theta)]$, as $g=$ $V U V^{\dagger}$, where

$$
\begin{aligned}
& U=e^{-i \frac{\gamma^{\prime}}{2}[\cos (\alpha) Z+\sin (\alpha) X]}, \\
& V=e^{-i \frac{\alpha^{\prime}}{2}[\cos (\psi) Z+\sin (\psi) X]} .
\end{aligned}
$$

Our second goal is to derive the values of $\alpha^{\prime}$ and $\psi$ given in Eq. (11). In Eq. (9a) we take $\gamma^{\prime}=2 \pi+\gamma$. However, the results of this proof do not depend on specific choice of $\gamma^{\prime}$, aside from implicit dependence hidden in $\alpha$ or other parameters.

Defining the adjoint group action as $\operatorname{Ad}_{g}(B) \equiv g B g^{-1}$ and using the fact that

$$
\operatorname{Ad}_{A}\left(e^{B}\right)=e^{A B A^{\dagger}}
$$

for any unitary $A$ and operator $B$, and that

$$
\operatorname{Ad}_{e^{i \theta Y / 2}}(Z)=\cos (\theta) Z+\sin (\theta) X,
$$

and similar identities obtained by cycling $X, Y$, and $Z$, we may rewrite

$$
\begin{aligned}
& U=\operatorname{Ad}_{e^{i \alpha Y / 2}}\left(e^{-i \frac{\gamma^{\prime}}{2} Z}\right), \\
& V=\operatorname{Ad}_{e^{i \psi Y / 2}}\left(e^{-i \frac{\alpha^{\prime}}{2} Z}\right) .
\end{aligned}
$$

Then setting $u=\alpha-\psi$, we see that

$$
\begin{aligned}
V U V^{\dagger} & =\operatorname{Ad}_{e^{i \psi Y / 2}}\left(\operatorname{Ad}_{e^{-i \alpha^{\prime} Z / 2}}\left[\operatorname{Ad}_{e^{i(\alpha-\psi) Y / 2}}\left(e^{-i \frac{\gamma^{\prime}}{2} Z}\right)\right]\right) \\
& =\operatorname{Ad}_{e^{i \psi Y / 2}}\left(\operatorname{Ad}_{e^{-i \alpha^{\prime} Z / 2}}\left(e^{-i \frac{\gamma^{\prime}}{2}(\cos (u) Z+\sin (u) X)}\right)\right) \\
& =\operatorname{Ad}_{e^{i \psi Y / 2}} e^{-i \frac{\gamma^{\prime}}{2}\left\{\cos (u) Z+\sin (u)\left[\cos \left(\alpha^{\prime}\right) X-\sin \left(\alpha^{\prime}\right) Y\right]\right\}} \\
& =e^{-i \frac{\gamma^{\prime}}{2}\left(v_{x} X+v_{y} Y+v_{z} Z\right)}
\end{aligned}
$$

where in the final line

$$
\begin{aligned}
& v_{x}=\cos (u) \sin (\psi)+\sin (u) \cos \left(\alpha^{\prime}\right) \cos (\psi), \\
& v_{y}=-\sin (u) \sin \left(\alpha^{\prime}\right), \\
& v_{z}=\cos (u) \cos (\psi)-\sin (u) \cos \left(\alpha^{\prime}\right) \sin (\psi) .
\end{aligned}
$$

Using the representation of the desired gate $g$ as in Eq. (E1), this gives a system of three equations: $v_{x}=r_{x}$,

$$
\begin{aligned}
\sin (\theta) \cos (\phi) & =\cos (u) \sin (\psi)+\sin (u) \cos \left(\alpha^{\prime}\right) \cos (\psi) \\
& =\sin (\alpha)+\sin (u) \cos (\psi)\left[\cos \left(\alpha^{\prime}\right)-1\right] ;
\end{aligned}
$$

$v_{y}=r_{y}$,

$$
\sin (\theta) \sin (\phi)=-\sin (u) \sin \left(\alpha^{\prime}\right)^{\prime} ;
$$

and $v_{z}=r_{z}$,

$$
\begin{aligned}
\cos (\theta) & =\cos (u) \cos (\psi)-\sin (u) \cos \left(\alpha^{\prime}\right) \sin (\psi) \\
& =\cos (\alpha)-\sin (u) \sin (\psi)\left[\cos \left(\alpha^{\prime}\right)-1\right]
\end{aligned}
$$

The existence of a solution of these three equations for $\alpha$, $\alpha^{\prime}$, and $\psi$ proves that $g$ can be written as $V U V^{\dagger}$ as claimed.

Equation (E9) immediately reduces to

$$
\sin \left(\alpha^{\prime}\right)=-\frac{\sin (\theta) \sin (\phi)}{\sin (u)},
$$


while Eqs. (E8) and (E10) can be combined into

$$
\tan (\psi)=\frac{\cos (\alpha)-\cos (\theta)}{\sin (\theta) \cos (\phi)-\sin (\alpha)} .
$$

Thus, we have the values of $\alpha^{\prime}$ and $\psi$ given in Eq. (11), up to a choice of the range of $\tan ^{-1}$ and $\sin ^{-1}$.

\section{APPENDIX F：TWO-QUBIT COUPLING DECOMPOSITION}

In this Appendix we derive the decomposition given in Eq. (17), and solve for the necessary $t, b$, and $\beta$ in terms of $C$. The decomposition in Appendix F 1 relies on an auxiliary decomposition, which is derived in Appendix F 2.

\section{Deriving Eq. (17)}

Pairwise coupling [Eq. (14)] allows use of the following two-qubit decomposition between qubits $i$ and $j$, derived in Appendix F 2:

$$
\begin{aligned}
& e^{-i t\left[a\left(X_{i}+X_{j}\right)+b Z_{i} Z_{j}\right]} \\
& \quad=e^{-i \beta\left(X_{i}+X_{j}\right)} e^{-i D_{1} X_{i} X_{j}} e^{-i D_{2} Y_{i} Y_{j}} e^{-i D_{3} Z_{i} Z_{j}} e^{-i \beta\left(X_{i}+X_{j}\right)}
\end{aligned}
$$

with

$$
\begin{aligned}
D_{1} & =0, \\
D_{2} & =\frac{1}{2}(b t-\omega), \\
D_{3} & =\frac{1}{2}(b t+\omega), \\
\omega & =\sin ^{-1}\left(\frac{b}{\sqrt{4 a^{2}+b^{2}}} \sin \left(t \sqrt{4 a^{2}+b^{2}}\right)\right), \\
\beta & =\frac{s}{4} \cos ^{-1}\left(\cos \left(t \sqrt{4 a^{2}+b^{2}}\right) \sec (\omega)\right)+\frac{\pi}{2},
\end{aligned}
$$

where $s=\operatorname{sign}\left[a \sin \left(\sqrt{4 a^{2}+b^{2}} t\right)\right]$ and we pick $\omega \in\left[-\frac{\pi}{2}, \frac{\pi}{2}\right]$ and $\beta \in[0, \pi]$. Uncoupled qubits have $w_{i j}=0 \forall j$, and simply experience $e^{-i a t X}$, as shown in Fig. 3 .

We restrict to the pure $Z Z$ coupling of Eq. (13) between qubits $i$ and $j$ by requiring that $D_{2}=0$ [to cancel the undesired $Y_{i} Y_{j}$ term in Eq. (F1b)], and thus that

$$
b t=\omega=D_{3} \equiv D .
$$

For coupled pairs, we take $b \neq 0$ and solve for $t$ in terms of $D$. Namely, substituting Eq. (F3) into (F2d) we obtain

$$
\operatorname{sinc}(D)=\operatorname{sinc}\left(\sqrt{4 a^{2} t^{2}+D^{2}}\right),
$$

where $\operatorname{sinc}(x) \equiv \sin (x) / x$.

Achieving the desired magnitude of coupling in Eq. (13) up to an overall phase requires that $D=C \bmod \pi$. For every value of $C \in[0, \pi]$, there exists a $k \in\{0,1,2,3\}$ such that the equation

$$
\operatorname{sinc}\left[\sqrt{x^{2}+(C+k \pi)^{2}}\right]=\operatorname{sinc}(C+k \pi)
$$

has a solution for some real $x$ [where $x=2 a t$ in Eq. (F4)]. This equation is transcendental and cannot be solved analytically. However, for a given pair of $(C, k)$, numerical methods can approximately solve Eq. (F5) in the domain $x>0$ or determine that no solution exists. We checked the solvability of Eq. (F5) for $C \in[0, \pi]$ and $k=0,1,2,3$ to determine the

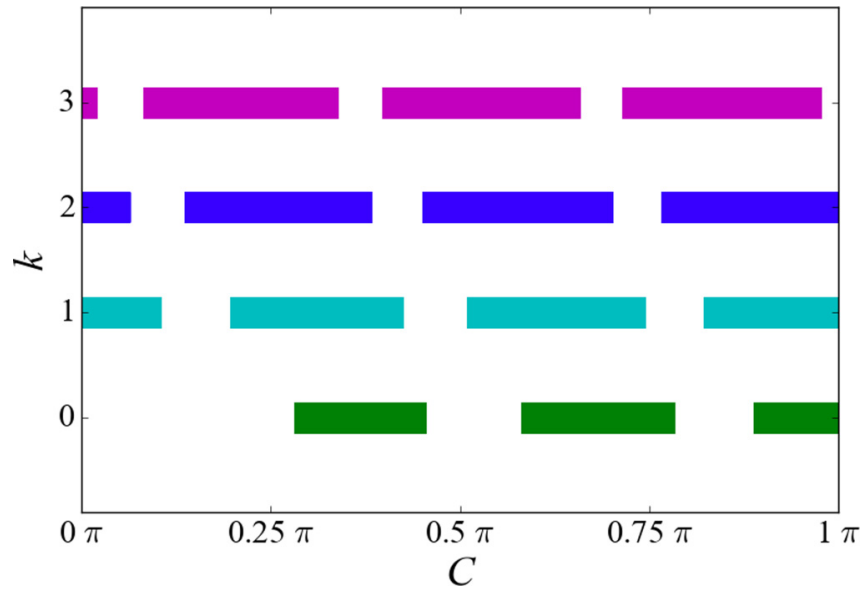

FIG. 10. Values of $C$ and $k$ for which Eq. (F5) can be solved for $x>0$. Color is included for visibility. For each value of $C$, at least one value of $k$ leads to a solution.

minimum range of $k$ needed to make Eq. (F5) solvable for all $C$. The results of this numerical determination are plotted in Fig. 10, from which one can see that $k \in\{0,1,2,3\}$ is sufficient. As a result, for any $C \in[0, \pi]$, we can pick this value of $k$ and corresponding numerical solution $t$, and set $b=D t^{-1}=(C+k \pi) t^{-1}$.

\section{Auxiliary decomposition Eq. (F1)}

The form of the decomposition in Eq. (F1) is motivated by Ref. [43]; any $U \in \mathrm{SU}(4)$ can be decomposed as

$$
U=k_{1} e^{-i\left[D_{1}(X \otimes X)+D_{2}(Y \otimes Y)+D_{3}(Z \otimes Z)\right]} k_{2},
$$

where $k_{1}, k_{2} \in \mathrm{SU}(2) \otimes \mathrm{SU}(2)$ and $D_{1}, D_{2}, D_{3} \in \mathbb{R}$. To write $U_{i j}=e^{-i t\left[a\left(X_{i}+X_{j}\right)+b Z_{i} Z_{j}\right]}$ in this form we first diagonalize $h_{i j}=$ $a\left(X_{i}+X_{j}\right)+b Z_{i} Z_{j}$ and obtain its eigenvalues $\left(e_{i}\right)$ and eigenstates $\left(\left|e_{i}\right\rangle\right)$. Then we can obtain $U_{i j}$ as $\sum_{i} e^{-i t e_{i}}\left|e_{i}\right\rangle\left\langle e_{i}\right|$. If we decompose $U_{i j}$ in the Pauli basis, we can show that it has the following form:

$$
\begin{aligned}
U_{i j}= & P_{00} I+P_{01} X_{j}+P_{10} X_{i}+P_{11} X_{i} X_{j} \\
& +P_{22} Y_{i} Y_{j}+P_{33} Z_{i} Z_{j},
\end{aligned}
$$

with other terms equal to zero. From this form we see that we do not need to consider the most general form of a single-qubit gate for $k_{1}$ and $k_{2}$. Instead we start from the following ansatz, which we will show to be sufficient:

$$
e^{-i \beta\left(X_{i}+X_{j}\right)} e^{-i D_{1} X_{i} X_{j}} e^{-i D_{2} Y_{i} Y_{j}} e^{-i D_{3} Z_{i} Z_{j}} e^{-i \beta\left(X_{i}+X_{j}\right)} .
$$

With this ansatz, the problem becomes solving the following equality:

$$
\begin{aligned}
& e^{-i t\left[a\left(X_{i}+X_{j}\right)+b Z_{i} Z_{j}\right]} \\
& \quad=e^{-i \beta\left(X_{i}+X_{j}\right)} e^{-i D_{1} X_{i} X_{j}} e^{-i D_{2} Y_{i} Y_{j}} e^{-i D_{3} Z_{i} Z_{j}} e^{-i \beta\left(X_{i}+X_{j}\right) .}
\end{aligned}
$$

This requires solving 16 coupled equations, each for one element of a $4 \times 4$ matrix. To simplify the task we represent both sides of Eq. (F9) in the "magic basis" defined in Ref. [44] as 


$$
\begin{aligned}
& \left|\phi_{1}\right\rangle=\frac{1}{\sqrt{2}}(|00\rangle+|11\rangle), \quad\left|\phi_{2}\right\rangle=\frac{i}{\sqrt{2}}(|00\rangle-|11\rangle), \\
& \left|\phi_{3}\right\rangle=\frac{1}{\sqrt{2}}(|01\rangle-|10\rangle), \quad\left|\phi_{4}\right\rangle=\frac{i}{\sqrt{2}}(|01\rangle+|10\rangle) .
\end{aligned}
$$

(F10b)

In this basis both matrices are sparse, and can be equated term by term to solve for the given parameters. Also, the nonlocal part of the right-hand side of Eq. (F9b), $e^{-i D_{1} X_{i} X_{j}} e^{-i D_{2} Y_{i} Y_{j}} e^{-i D_{3} Z_{i} Z_{j}}$, is diagonal in the magic basis. The following matrix changes the basis from the computational basis to the magic basis:

$$
Q=\frac{1}{\sqrt{2}}\left[\begin{array}{cccc}
1 & i & 0 & 0 \\
0 & 0 & 1 & i \\
0 & 0 & -1 & i \\
1 & -i & 0 & 0
\end{array}\right] .
$$

Using $Q$ we can write any matrix $U \in \mathrm{SU}(4)$ in the magic basis as

$$
U_{\text {mag }}=\sum_{i, j}\left(Q^{\dagger} U Q\right)_{j i}\left|\phi_{j}\right\rangle\left\langle\phi_{i}\right| .
$$

The ansatz defined in Eq. (F9b) takes the following form in this basis:

$$
\left[\begin{array}{cccc}
u_{11} & 0 & 0 & u_{14} \\
0 & u_{22} & 0 & 0 \\
0 & 0 & u_{33} & 0 \\
u_{41} & 0 & 0 & u_{44}
\end{array}\right]
$$

where

$$
\begin{aligned}
u_{11}= & \frac{1}{2} e^{-i\left(D_{1}+D_{2}+D_{3}\right)}\left[e^{2 i D_{3}}(\cos (4 \beta)-1]\right. \\
& \left.+e^{2 i D_{2}}[\cos (4 \beta)+1)\right], \\
u_{14}= & e^{-i D_{1}} \sin (4 \beta) \cos \left(D_{2}-D_{3}\right), \\
u_{22}= & e^{i\left(D_{1}-D_{2}-D_{3}\right)}, \\
u_{33}= & e^{i\left(D_{1}+D_{2}+D_{3}\right)}, \\
u_{41}= & -e^{-i D_{1}} \sin (4 \beta) \cos \left(D_{2}-D_{3}\right), \\
u_{44}= & \frac{1}{2} e^{-i\left(D_{1}+D_{2}+D_{3}\right)}\left[e^{2 i D_{2}}(\cos (4 \beta)-1]\right. \\
& \left.+e^{2 i D_{3}}[\cos (4 \beta)+1)\right] .
\end{aligned}
$$

The unitary defined in Eq. (F9a) takes the same form as shown in Eq. (F13) in the magic basis, such that

$$
\begin{aligned}
& u_{11}=\cos \left(t \sqrt{4 a^{2}+b^{2}}\right)-\frac{i b \sin \left(t \sqrt{4 a^{2}+b^{2}}\right)}{\sqrt{4 a^{2}+b^{2}}}, \\
& u_{14}=\frac{2 a \sin \left(t \sqrt{4 a^{2}+b^{2}}\right)}{\sqrt{4 a^{2}+b^{2}}}, \\
& u_{22}=e^{-i b t}, \\
& u_{33}=e^{i b t}, \\
& u_{41}=-\frac{2 a \sin \left(t \sqrt{4 a^{2}+b^{2}}\right)}{\sqrt{4 a^{2}+b^{2}}}, \\
& u_{44}=\cos \left(t \sqrt{4 a^{2}+b^{2}}\right)+\frac{i b \sin \left(t \sqrt{4 a^{2}+b^{2}}\right)}{\sqrt{4 a^{2}+b^{2}}} .
\end{aligned}
$$

Equating Eqs. (F14) and (F15) line by line, we obtain Eq. (F2).

\section{APPENDIX G: IMPLEMENTING SWAP WITH THE ALTERNATING $X$-FIELD MODEL}

In this Appendix we show in detail how we can implement the SWAP gate in seven layers using the alternating $X$-field method. In this method, instead of implementing the SWAP gate as three CNOT gates as in Fig. 8(a), we use different building blocks: $Z Z$-coupling layers with nonzero $X$ fields on either only the even or only the odd qubits, as shown in Fig. 9. First we start from one of these coupling layers with $X$ fields on only the even qubits. We can write the action of this layer as

$$
U=\prod_{i=\text { odd }} e^{-i t\left(a X_{i+1}+b Z_{i} Z_{i+1}\right)}
$$

for appropriately chosen $b$. Consider just the two-qubit gate acting on the first and second qubits; it has the following block-diagonal structure:

$$
U_{12}=e^{-i t\left(a X_{2}+b Z_{1} Z_{2}\right)}=\left[\begin{array}{cc}
U_{1} & \mathbf{0} \\
\mathbf{0} & U_{2}
\end{array}\right],
$$

where $\mathbf{0}$ is the $2 \times 2$ zero matrix, and we can further decompose

$$
\begin{aligned}
& U_{1}=e^{i \alpha Y} e^{-i \gamma X} e^{-i \alpha Y}, \\
& U_{2}=e^{-i \alpha Y} e^{-i \gamma X} e^{i \alpha Y},
\end{aligned}
$$

where

$$
\begin{aligned}
& \alpha=\frac{1}{2} \cos ^{-1}\left(\frac{a}{\sqrt{a^{2}+b^{2}}}\right), \\
& \gamma=t \sqrt{a^{2}+b^{2}} .
\end{aligned}
$$

If we set $b=a$ and $t=\frac{\pi}{2 \sqrt{2} a}$, then $U_{1}=-i W$, and $U_{1}^{\dagger} U_{2}=$ $\tilde{Y}$, where $\tilde{Y}=Z X=\left[\begin{array}{cc}0 & 1 \\ -1 & 0\end{array}\right]$. Thus, for this choice of $b$ and $t$,

$$
U_{12}=-i(I \otimes W) C \tilde{Y}_{12},
$$

where $C \tilde{Y}_{12}$ is the controlled $\tilde{Y}$ gate. Similar to the $Z Z$ coupling gates (recall the discussion in Sec. IVB), this operation leads to a net $X$ rotation on uncoupled qubits which still experience an $X$ field, which then must be undone by an extra single-qubit unitary following the SWAP layer.

Defining the above building block $U_{12}$, the total circuit required for a SWAP gate will be

$$
\begin{aligned}
C & =U_{12} W_{1} W_{2} U_{21} U_{12} W_{2} \\
& =i\left(W_{2} C \tilde{Y}_{12}\right) W_{1} W_{2}\left(W_{1} C \tilde{Y}_{21}\right)\left(W_{2} C \tilde{Y}_{12}\right) W_{2} .
\end{aligned}
$$

The product of $W_{1} W_{2}$ needs just one effective single-qubit gate layer in total, and any effect on uncoupled qubits can be incorporated into the extra single-qubit unitary $e^{-i \gamma X_{\mathcal{S}}}$ on these qubits following the SWAP layer. Since the last $W_{2}$ requires three single-qubit gate layers, the total number of required layers for implementing the above decomposition is seven.

Defining $\tilde{\bar{Y}}=-\tilde{Y}=X Z$ and using the equality $W_{2} C \tilde{Y}_{12}=$ $C \tilde{\bar{Y}}_{12} W_{2}$, we can simplify the above expression as below:

$$
\begin{aligned}
C & =i W_{2} C \tilde{Y}_{12} W_{1} W_{2} W_{1} C \tilde{Y}_{21} W_{2} C \tilde{Y}_{12} W_{2} \\
& =i C \tilde{\bar{Y}}_{12} W_{2} W_{1} W_{2} W_{1} C \tilde{Y}_{21} C \tilde{\bar{Y}}_{12} W_{2} W_{2}
\end{aligned}
$$




$$
\begin{aligned}
& =i C \tilde{\bar{Y}}_{12} C \tilde{Y}_{21} C \tilde{\bar{Y}}_{12} \\
& =i C X_{12} C Z_{12} C Z_{21} C X_{21} C X_{12} C Z_{12} \\
& =i C X_{12} C X_{21} C X_{12} C Z_{12} \\
& =i \operatorname{SWAP}_{12} C Z_{12} \\
& =i C Z_{21} \operatorname{SWAP}_{12} .
\end{aligned}
$$

This is the required SWAP gate up to $C Z_{21}$. This unwanted $C Z_{21}$ can be written as a product of single-qubit $Z$-diagonal gates and $Z Z$ couplings, and can in fact be incorporated into the existing single-qubit and coupling gates already in the overall circuit, simply by shifting the values of $w_{12}, v_{1}$, and $v_{2}$ by $\frac{\pi}{4}$. These $v_{i}$ and $w_{i j}$ parameters are chosen from a uniform distribution over the full range of multiples of $\frac{\pi}{8} \bmod \pi$, so the shift by $\frac{\pi}{4}$ does not affect the distribution. This makes the $C Z$ gate effectively free to include.
[1] J. Preskill, Quantum computing in the nisq era and beyond, Quantum 2, 79 (2018).

[2] D. Deutsch, Quantum theory, the church-turing principle and the universal quantum computer, Proc. R. Soc. London, Ser. A 400, 97 (1985).

[3] D. P. DiVincenzo, Two-bit gates are universal for quantum computation, Phys. Rev. A 51, 1015 (1995).

[4] S. Lloyd, Almost any Quantum Logic Gate is Universal, Phys. Rev. Lett. 75, 346 (1995).

[5] S. Lloyd, Quantum approximate optimization is computationally universal, arXiv:1812.11075.

[6] R. Raussendorf, Quantum computation via translation-invariant operations on a chain of qubits, Phys. Rev. A 72, 052301 (2005).

[7] J. Preskill, Quantum computing and the entanglement frontier, arXiv:1203.5813.

[8] M. J. Bremner, A. Montanaro, and D. J. Shepherd, AverageCase Complexity versus Approximate Simulation of Commuting Quantum Computations, Phys. Rev. Lett. 117, 080501 (2016).

[9] M. J. Bremner, R. Jozsa, and D. J. Shepherd, Classical simulation of commuting quantum computations implies collapse of the polynomial hierarchy, Proc. R. Soc., Ser. A 467, 459 (2011).

[10] E. Farhi and A. W. Harrow, Quantum supremacy through the quantum approximate optimization algorithm, arXiv: 1602.07674.

[11] S. Aaronson and L. Chen, Complexity-theoretic foundations of quantum supremacy experiments, in CCC '17: Proceedings of the 32nd Computational Complexity Conference (Schloss Dagstuhl-Leibniz-Zentrum fuer Informatik, Dagstuhl, Germany, 2017).

[12] A. W. Harrow and A. Montanaro, Quantum computational supremacy, Nature (London) 549, 203 (2017).

[13] A. Bouland, B. Fefferman, C. Nirkhe, and U. Vazirani, On the complexity and verification of quantum random circuit sampling, Nat. Phys. 15, 159 (2018).

[14] J. Bermejo-Vega, D. Hangleiter, M. Schwarz, R. Raussendorf, and J. Eisert, Architectures for Quantum Simulation Showing a Quantum Speedup, Phys. Rev. X 8, 021010 (2018).

[15] J. Haferkamp, D. Hangleiter, A. Bouland, B. Fefferman, J. Eisert, and J. Bermejo-Vega, Closing Gaps of a Quantum Advantage with Short-Time Hamiltonian Dynamics, Phys. Rev. Lett. 125, 250501 (2020).

[16] F. Arute, K. Arya, R. Babbush, D. Bacon, J. C. Bardin, R. Barends, R. Biswas, S. Boixo, F. G. S. L. Brandao, D. A. Buell, B. Burkett, Yu Chen, Z. Chen, B. Chiaro, R. Collins, W. Courtney, A. Dunsworth, E. Farhi, B. Foxen, A. Fowler et al., Quantum supremacy using a programmable superconducting processor, Nature (London) 574, 505 (2019).
[17] A. Zlokapa, S. Boixo, and D. Lidar, Boundaries of quantum supremacy via random circuit sampling, arXiv:2005.02464.

[18] D. Aharonov, D. Gottesman, S. Irani, and J. Kempe, The power of quantum systems on a line, Commun. Math. Phys. 287, 41 (2009).

[19] H. Meier, R. T. Brierley, A. Kou, S. M. Girvin, and L. I. Glazman, Signatures of quantum phase transitions in the dynamic response of fluxonium qubit chains, Phys. Rev. B 92, 064516 (2015).

[20] C. Neill, P. Roushan, K. Kechedzhi, S. Boixo, S. V. Isakov, V. Smelyanskiy, A. Megrant, B. Chiaro, A. Dunsworth, K. Arya, R. Barends, B. Burkett, Y. Chen, Z. Chen, A. Fowler, B. Foxen, M. Giustina, R. Graff, E. Jeffrey, T. Huang et al., A blueprint for demonstrating quantum supremacy with superconducting qubits, Science 360, 195 (2018).

[21] J. Q. You, Xuedong $\mathrm{Hu}, \mathrm{S}$. Ashhab, and F. Nori, Lowdecoherence flux qubit, Phys. Rev. B 75, 140515 (2007).

[22] R. Harris, J. Johansson, A. J. Berkley, M. W. Johnson, T. Lanting, Siyuan Han, P. Bunyk, E. Ladizinsky, T. Oh, I. Perminov, E. Tolkacheva, S. Uchaikin, E. M. Chapple, C. Enderud, C. Rich, M. Thom, J. Wang, B. Wilson, and G. Rose, Experimental demonstration of a robust and scalable flux qubit, Phys. Rev. B 81, 134510 (2010).

[23] F. Yan, S. Gustavsson, A. Kamal, J. Birenbaum, A. P. Sears, D. Hover, T. J. Gudmundsen, D. Rosenberg, G. Samach, S. Weber, J. L. Yoder, T. P. Orlando, J. Clarke, A. J. Kerman, and W. D. Oliver, The flux qubit revisited to enhance coherence and reproducibility, Nat. Commun. 7, 12964 EP (2016).

[24] J. A. Grover, J. I. Basham, A. Marakov, S. M. Disseler, R. T. Hinkey, M. Khalil, Z. A. Stegen, T. Chamberlin, W. DeGottardi, D. J. Clarke, J. R. Medford, J. D. Strand, M. J. A. Stoutimore, S. Novikov, D. G. Ferguson, D. Lidar, K. M. Zick, and A. J. Przybysz, Fast, lifetime-preserving readout for high-coherence quantum annealers, PRX Quantum 1, 020314 (2020).

[25] E. Mozgunov and D. A. Lidar, Quantum adiabatic theorem for unbounded hamiltonians, with applications to superconducting circuits, arXiv:2011.08116.

[26] E. Farhi, J. Goldstone, and S. Gutmann, A quantum approximate optimization algorithm, arXiv:1411.4028.

[27] S. C. Benjamin, Quantum Computing without Local Control of Qubit-Qubit Interactions, Phys. Rev. Lett. 88, 017904 (2001).

[28] M. E. S. Morales, J. D. Biamonte, and Z. Zimborás, On the universality of the quantum approximate optimization algorithm, Quantum Inf. Proc. 19, 1 (2020).

[29] D. C. McKay, C. J. Wood, S. Sheldon, J. M. Chow, and J. M. Gambetta, Efficient $z$ gates for quantum computing, Phys. Rev. A 96, 022330 (2017). 
[30] A. Barenco, C. H. Bennett, R. Cleve, D. P. DiVincenzo, N. Margolus, P. Shor, T. Sleator, J. A. Smolin, and H. Weinfurter, Elementary gates for quantum computation, Phys. Rev. A 52, 3457 (1995).

[31] M. A. Nielsen and I. L. Chuang, Quantum Computation and Quantum Information (Cambridge University Press, Cambridge, 2010).

[32] C. M. Dawson and M. A. Nielsen, The solovay-kitaev algorithm, Quantum Inf. Comput. 6, 81 (2006).

[33] D. Hangleiter, J. Bermejo-Vega, M. Schwarz, and J. Eisert, Anticoncentration theorems for schemes showing a quantum speedup, Quantum 2, 65 (2018).

[34] M. J. Bremner, A. Montanaro, and D. J. Shepherd, Achieving quantum supremacy with sparse and noisy commuting quantum computations, Quantum 1, 8 (2017).

[35] V. G. Vizing, The chromatic class of a multigraph, Cybern. Syst. Anal. 1, 32 (1965).

[36] H.-S. Zhong, H. Wang, Y.-H. Deng, M.-C. Chen, L.-C. Peng, Y.-H. Luo, J. Qin, D. Wu, X. Ding, Y. Hu, P. Hu, X.-Y. Yang, W.-J. Zhang, H. Li, Y. Li, X. Jiang, L. Gan, G. Yang, L. You, Z. Wang, Li Li, N.-L. Liu, Chao-Yang Lu, and J.-W. Pan,
Quantum computational advantage using photons, Science 370, 1460 (2020).

[37] S. Aaronson and A. Arkhipov, The computational complexity of linear optics, in Proceedings of the Forty-Third Annual ACM Symposium on Theory of Computing (Association for Computing Machinery, San Jose, California, USA, 2011), pp. 333-342.

[38] S. Toda, Pp is as hard as the polynomial-time hierarchy, SIAM J. Comput. 20, 865 (1991).

[39] M. Szegedy, What do QAOA energies reveal about graphs? arXiv:1912.12277.

[40] R. Bhatia, Matrix Analysis, Number 169 in Graduate Texts in Mathematics (Springer, New York, 1997)

[41] D. A. Lidar, P. Zanardi, and K. Khodjasteh, Distance bounds on quantum dynamics, Phys. Rev. A 78, 012308 (2008).

[42] Quantum Error Correction, edited by D. A. Lidar and T. A. Brun (Cambridge University Press, Cambridge, UK, 2013).

[43] J. Zhang, J. Vala, S. Sastry, and K. Birgitta Whaley, Geometric theory of nonlocal two-qubit operations, Phys. Rev. A 67, 042313 (2003).

[44] S. Hill and W. K. Wootters, Entanglement of a Pair of Quantum Bits, Phys. Rev. Lett. 78, 5022 (1997). 